\title{
WAVE OPERATORS AND SIMILARITY FOR GENERATORS OF SEMIGROUPS IN BANACH SPACES $\left(^{1}\right)$
}

\author{
BY \\ SUE-CHIN LIN
}

1. Introduction. This paper is concerned with perturbation and similarity of linear operators $T$ in a Banach space $X$ whose resolvent sets contain a half plane and such that the operator $-i T$ (or $i T$ ) generates strongly continuous semigroups of bounded operators of $\mathfrak{B}(X)$.

During recent decades many features on the perturbation of linear operators have commanded a considerable interest and have been studied extensively. Among them, the theory on perturbation of continuous spectra, originated by K. O. Friedrichs [1], [2], was pursued by many authors, such as Rosenblum [3], Kato [4], [5], [6], Ladyženskaja and Faddeev [7], Schwartz [8], Kuroda [9], [10], de Branges [11], Rejto [12], Birman [13], [14] and others. Generally speaking, approaches to the problem of the similarity (or unitary equivalence) between a perturbed operator $T(\kappa)$, formally given by $T+\kappa V$, and the unperturbed operator $T$ are classified as "stationary" or "time-dependent" according to whether the intertwining operators (nonsingular, partial isometric or unitary) are constructed from the resolvents or from the semigroups (groups or unitary groups) of the operators. In his recent paper $[6, \S 3] \mathrm{T}$. Kato obtained some results on the similarity of certain generators of strongly continuous groups of bounded operators in Hilbert spaces. The basic assumptions in [6] are that the resolvent set of the unperturbed operator $T$ (not necessarily symmetric, nor bounded) contains both the upper and the lower half planes $\Omega_{ \pm}=\{z \mid \operatorname{Im} z \gtrless 0\}$ and that $-i T$ is the infinitesimal generator of a strongly continuous group of operators of $\mathfrak{B}(H)$ with type zero ( $H$ Hilbert space). Our results in this paper are generalizations of [6] in two respects. First we try to extend Kato's work [6] from Hilbert spaces to more general Banach spaces. Second we consider the perturbation problem in which the resolvent set of the unperturbed operator $T$ may contain only a half plane $\Omega_{+}$ (or $\Omega_{-}$) and further $-i T$ (or $i T$ ) generate a strongly continuous semigroup.

The method used in this investigation is of the time-dependent nature. The main results here can be formulated briefly as follows. Let $X, Y$ be complex reflexive separable Banach spaces. Let $T, \hat{T}$ and $V$ be operators (in general unbounded) in $X$

Received by the editors January 15, 1968 and, in revised form, May 13, 1968.

( $\left.{ }^{1}\right)$ This work contains part of the author's doctoral dissertation submitted to the University of California at Berkeley. The author wishes to express his sincere gratitude to Professor Tosio Kato for his patient guidance and fruitful instruction. Part of this work was supported by NSF grant GP-1611 and AFOSR grant 553-64. 
with resolvent sets $\rho(T) \supset \Omega_{+}, \rho(\hat{T}) \supset \Omega_{-}$and the operators -iT, $i \hat{T}$ generate, respectively, the strongly continuous semigroups $\left\{e^{-i t T}\right\}_{0 \leqq t<\infty}$ and $\left\{e^{i t \hat{T}}\right\}_{0 \leqq t<\infty}$ of bounded operators of $\mathfrak{B}(X)$ of class $C_{0}$ with type zero. We assume that $T \subset \hat{T}$ and that the operator $V$ can be factored formally as the product $B A$ of two operators $A$ from $X$ to $Y$ and $B$ from $Y$ to $X$. We assume further that $A$ and $B$ behave smoothly (see Definition 2.4 ) with respect to $T$ and $\hat{T}$. Under these smoothness conditions on the disturbance $V$, the perturbed operator $T(\kappa)$ and $\hat{T}(\kappa)$, formally given by $T+\kappa B A$ and $\hat{T}+\kappa B A$, respectively, are similar to $T$ and $\hat{T}$, respectively. These similarities are implemented by an intertwining operator $W(\kappa)$ (called the wave operator) constructed explicitly from the semigroups.

Applications are made to "gentle perturbations of second kind", originated by K. O. Friedrichs [2, §12], [15], in which $T=-i d / d x$ and the disturbances are integral operators with kernel $k(x, y)$ satisfying the condition

$$
\int_{0}^{\infty} \int_{0}^{\infty}|k(x, y)| d x d y<\infty
$$

With the help of a factorization for the kernel of such disturbances, we are able to handle the second gentle perturbation completely by our method. This answers partly the question raised in Kato [6] about the relationship between our smoothness conditions on $V$ and the gentleness conditions on $V$ given by Friedrichs.

2. Preliminaries. Let $X$ and $Y$ designate separable reflexive Banach spaces of positive dimensions over the complex field with their dual spaces (Banach dual) $X^{\prime}$ and $Y^{\prime}$, respectively. The symbol $\|\cdot\|$ will denote the norms of both $X, Y$ and their duals. The value of the functional $f \in X^{\prime}$ at the point $x \in X$ is denoted by $\langle x, f\rangle$. The Banach adjoint of an operator $A$ (throughout this paper by operator we shall mean linear operator) is denoted by $A^{\prime}$. The class of all closed operators with domain in $X$ and range in $Y$ will be denoted by $\mathfrak{C}(X, Y) . \mathfrak{B}(X, Y)$ stands for all bounded operators with domain the whole range of $X$ and the range in $Y$. $\mathfrak{S}_{0}(X, Y)$ is the set of all $T \in \mathfrak{E}(X, Y)$ with domain $D(T)$ dense in $X$. We shall write $\mathfrak{S}(X)$ for $\mathfrak{s}(X, X), \mathfrak{\subseteq}_{0}(X)$ for $\mathfrak{\mho}_{0}(X, X)$ and $\mathfrak{B}(X)$ for $\mathfrak{B}(X, X)$.

Definition 2.1. We denote by $\mathfrak{G S}_{+}(X)$ the set of all $T \in \mathfrak{E}_{0}(X)$ with $\rho(T) \supset \Omega_{+}$ such that $-i T$ generates a strongly continuous semigroup $\{U(t)\}_{0 \leqq t<\infty}$ of operators of $\mathfrak{B}(X)$ of class $C_{0}$ with type zero (i.e. $\{U(t)\}_{0 \leqq t<\infty}$ is continuous in the strong operator topology of $\mathfrak{B}(X)$ and

$$
\lim _{t \rightarrow 0} U(t) u=u \text { for all } u \in X
$$

$$
\lim _{t \rightarrow \infty} e^{-\varepsilon t}\|U(t)\|=0
$$

for every $\varepsilon>0$. (Cf. Hille and Phillips [16].) We shall write $e^{-i t T}$ for $U(t)$. Similarly, we define the class $\mathscr{S}_{-}(X)$ as the set of all $T \in \mathfrak{S}_{0}(X)$ with $\rho(T) \supset \Omega_{-}$such that $i T$ generates a strongly continuous semigroup $\left\{e^{i t T}\right\}_{0 \leqq t<\infty}$ of class $C_{0}$ with type zero. 
We note that whenever $T \in \mathscr{S}_{+}(X)\left(B_{-}(X)\right)$ so does $T^{\prime}$, since our spaces are reflexive (cf. [16, Chapter XIV]).

Definition 2.2. Let $A \in \mathfrak{C}_{0}(X, Y)$. We introduce an extended definition of norm by setting $\|A u\|=\infty$ if $u \in X$ but $u \notin D(A)$.

This extended norm is only a symbolic notation, since $A u$ does not make sense if $u \notin D(A)$. Thus, if $B$ is another operator with its range contained in $X$, we use the notation $\|A B u\|$ only when $u \in D(B)$ so that $B u$ is well defined; then $\|A B u\|<\infty$ if $B u \in D(A)$ and $\|A B u\|=\infty$ otherwise.

Since $A \in \mathfrak{S}_{0}(X, Y)$ and $Y$ is reflexive, we have $A^{\prime} \in \mathfrak{S}_{0}\left(Y^{\prime}, X^{\prime}\right)$ (cf. [16, Theorem 2.11 .9$, p. 43]). It follows that the graph $G\left(A^{\prime}\right)$ of $A^{\prime}$ is a closed linear subspace of the separable Banach space $Y^{\prime} \times X^{\prime}$ (for both $Y^{\prime}$ and $X^{\prime}$ are separable) under the norm $\|\{u, v\}\|=\|u\|+\|v\|, \quad\{u, v\} \in Y^{\prime} \times X^{\prime} \quad$ (cf. Kato [17, pp. 164-165]). Thus $G\left(A^{\prime}\right)$ contains a countable dense subset $\left\{W_{n}\right\}, W_{n}=\left\{w_{n}, A^{\prime} w_{n}\right\}$. The set $\left\{w_{n}\right\}$ is clearly dense in $D\left(A^{\prime}\right)$ (because the projection from $G\left(A^{\prime}\right) \rightarrow D\left(A^{\prime}\right)$ is a bounded operator), and hence in $Y^{\prime}$. With this set $\left\{w_{n}\right\}$ at our disposal, we claim

LEMMA 2.3.

$$
\|A u\|=\sup _{n} \frac{\left|\left\langle u, A^{\prime} w_{n}\right\rangle\right|}{\left\|w_{n}\right\|}, \text { for all } u \in X, w_{n} \neq 0
$$

where the extended norm defined above is used.

Proof. Let

$$
\alpha=\sup _{n} \frac{\left|\left\langle u, A^{\prime} w_{n}\right\rangle\right|}{\left\|w_{n}\right\|}, \quad w_{n} \neq 0 .
$$

If $u \in D(A)$, it is easily seen that

$$
\|A u\|=\sup _{w \neq 0 ; w \in Y^{\prime}} \frac{|\langle A u, w\rangle|}{\|w\|}=\sup _{n ; w_{n} \neq 0} \frac{\left|\left\langle A u, w_{n}\right\rangle\right|}{\left\|w_{n}\right\|}=\alpha
$$

since $\left\{w_{n}\right\}$ is dense in $Y^{\prime}$. Hence $(2.1)$ holds.

On the other hand, if $u \in X$ is such that $\alpha<\infty$, then for any $v \in D\left(A^{\prime}\right), \varepsilon>0$, there exists $W_{n}$ such that

$$
\left\|W_{n}-\left\{v, A^{\prime} v\right\}\right\|<\varepsilon,
$$

which implies that $\left\|w_{n}-v\right\|<\varepsilon$ and $\left\|A^{\prime} w_{n}-A^{\prime} v\right\|<\varepsilon$. It follows, by a simple estimation, that

$$
\left|\left\langle u, A^{\prime} v\right\rangle\right|<\varepsilon(\|u\|+\alpha)+\alpha\|v\| .
$$

Since $\varepsilon$ is arbitrary, we thus have $\left|\left\langle u, A^{\prime} v\right\rangle\right| \leqq \alpha\|v\|$, for all $v \in D\left(A^{\prime}\right)$. This implies that $u \in D\left(A^{\prime \prime}\right)=D(A)$. Hence the lemma.

We have, by (2.1),

$$
\left\|A e^{-i t T} u\right\|=\sup _{n: w_{n} \neq 0} \frac{\left|\left\langle e^{-i t T} u, A^{\prime} w_{n}\right\rangle\right|}{\left\|w_{n}^{\prime}\right\|}, \text { for } u \in X .
$$


Since $e^{-i t T}$ is strongly continuous in $t$, the function $\left\langle e^{-i t T} u, A^{\prime} w_{n}\right\rangle$ is continuous in $t$ for each $n$; it follows that $\left\|A e^{-i t T} u\right\|$ is measurable in $t$ (measurable refers to Lebesgue measure throughout this paper, cf. Halmos [18, Theorem A, p. 84]).

Definition 2.4. Let $T \in \mathscr{S}_{+}(X), A \in \mathfrak{S}_{0}(X, Y)$ and $1<p<\infty$. We say that $A$ is $(p,+)$-smooth with respect to $T$, or simply $(T, p,+)$-smooth, if there exists a constant $M_{u}<\infty$ such that

$$
\int_{0}^{\infty}\left\|A e^{-i t T} u\right\|^{p} d t \leqq M_{u}^{p}, \quad \text { for each } u \in X .
$$

It follows from (2.3) that if $A$ is $(T, p,+)$-smooth, then for any $u \in X, e^{-i t T} u \in D(A)$ for a.e. $t$.

LeMmA 2.5. If $A$ is $(T, p,+)$-smooth, then the mapping $u \rightarrow A e^{-i t T} u, 0 \leqq t<\infty$, defines a bounded linear operator $S$ on $X$ to $\mathscr{Y}=L^{p}(0, \infty ; Y)$.

This lemma can be proved easily by using standard arguments on convergence in $L^{p}$ spaces and the closed graph theorem.

As a consequence of Lemma 2.5, whenever $A$ is $(T, p,+)$-smooth there exists a constant $M<\infty$ such that

$$
\int_{0}^{\infty}\left\|A e^{-i t T} u\right\|^{p} d t \leqq M^{p}\|u\|^{p}, \quad \text { for all } u \in X .
$$

We shall denote by $\|A\|_{\left(T, p_{0}+\right)}$ the infimum of all $M$ satisfying (2.4). We define smoothness of an operator $A \in \mathfrak{E}_{0}(X, Y)$ with respect to a $\hat{T} \in \mathfrak{S}_{-}(X)$ by replacing $-i$ and $T$ with $i$ and $\hat{T}$, respectively, throughout Definition 2.4 and Lemma 2.5, and arrive at

$$
\int_{0}^{\infty}\left\|A e^{i t} \hat{\imath}\right\|^{p} d t \leqq M^{p}\|u\|^{p}, \quad \text { for all } u \in X .
$$

Definition 2.6. Let $T \in \mathscr{S}_{+}(X)$ and $\hat{T} \in \mathscr{S}_{-}(X)$. If $T \subset \hat{T}$, then we say that the pair $(T, \hat{T}) \in \mathscr{S}_{ \pm}(X)$. If $A$ is $(T, p,+)$-smooth and $(\hat{T}, p,-)$-smooth with respect to a pair $(T, \hat{T}) \in \mathscr{B}_{ \pm}(X)$, then we say $A$ is $(T, \hat{T} ; p)$-smooth, and set

$$
\|A\|_{(T, \hat{T} ; p)}=\max \left\{\|A\|_{(T, p,+)},\|A\|_{(\hat{T}, p,-)}\right\} .
$$

Lemma 2.7. Let $T \in \mathfrak{B S}_{+}(X)$ and $B \in \mathfrak{C}_{0}(Y, X)$. Assume that $B^{\prime}$ is $\left(T^{\prime}, q,+\right)$ smooth, $1<q<\infty$. Then for each $f \in \mathscr{Y}=L^{p}(0, \infty ; Y)$ where $1 / p+1 / q=1$, there exists an $X$ valued function $F(t)$ on $(0, \infty)$ such that

$$
\langle F(t), v\rangle=\int_{0}^{t}\left\langle f(s), B^{\prime} e^{-i(t-s) T^{\prime}} v\right\rangle d s, \text { for } v \in X^{\prime} .
$$

Proof. For any $f \in \mathscr{Y}$ and $v \in X^{\prime}$ we have the following estimate

$$
\left|\int_{0}^{t}\left\langle f(s), B^{\prime} e^{-i(t-s) T^{\prime}} v\right\rangle d s\right| \leqq\|f\| \mathscr{Y}\left\|B^{\prime}\right\|_{\left(T^{\prime}, q,+\right)}\|v\| .
$$


Thus the map $v \rightarrow \int_{0}^{t}\left\langle f(s), B^{\prime} e^{-t(t-s) T^{\prime}} v\right\rangle d s$ defines a bounded linear functional on $X^{\prime}$. Hence for every $0<t<\infty$ there exists $F(t) \in X$ such that $\langle F(t), v\rangle$ $=\int_{0}^{t}\left\langle f(s), B^{\prime} e^{-i(t-s) T^{\prime}} v\right\rangle d s$. Moreover, $\|F(t)\| \leqq\|f\|_{\mathscr{Y}}\left\|B^{\prime}\right\|_{\left(T^{\prime}, q,+\right)}$ uniformly in $t$.

Definition 2.8. Let $T \in \mathscr{B S}_{+}(X), A \in \mathfrak{E}_{0}(X, Y)$ and $B \in \mathfrak{C}_{0}(Y, X)$. Assume that $A$ is $(T, p,+)$-smooth and $B^{\prime}$ is $\left(T^{\prime}, q,+\right)$-smooth. We define an operator, symbolically denoted by $A e^{-i(\cdot) T} B \theta$, in $\mathscr{Y}=L^{p}(0, \infty ; Y)$ as follows. For each $f \in \mathscr{Y}$, let $F(t)$ be the function defined in Lemma 2.7 associated with $f$. If the function $F(t)$ has the properties that (i) $F(t) \in D(A)$ for a.e. $t$ and (ii) $A F(t)=g(t)$, as a function of $t$, belongs to $\mathscr{Y}$, then we say that $f \in D\left(A e^{-i(\cdot) T} B \theta\right)$ and define $A e^{-i(\cdot) T} B \theta f=g$.

It should be noted that in general this domain of $A e^{-i(\cdot) T} B \theta$ may consist of the zero element alone.

Similarly, operator $A e^{i(\cdot) \hat{T}} B \theta$ can be defined in the same way for $\hat{T} \in \mathcal{E S}_{-}(X)$. We have

$$
\langle\hat{F}(t), v\rangle=\int_{0}^{t}\left\langle f(s), B^{\prime} e^{i(t-s) \hat{T}^{\prime}} v\right\rangle d s, \text { for } v \in X^{\prime}
$$

\section{Main results.}

THEOREM 3.1. Let $X, Y$ be complex reflexive separable Banach spaces. Let $(T, \hat{T}) \in \mathbb{S}_{ \pm}(X), A \in \mathfrak{C}_{0}(X, Y)$ and $B \in \mathfrak{E}_{0}(Y, X)$. Assume that $A$ is $(T, \hat{T} ; p)$-smooth and $B^{\prime}$ is $\left(\hat{T}^{\prime}, T^{\prime} ; q\right)$-smooth, where $1 / p+1 / q=1,1<p<\infty$. Furthermore, assume that $A e^{-i(\cdot) T} B \theta$ and $A e^{i(\cdot) \hat{T}} B \theta$ belong to $\mathfrak{B}(\mathscr{Y})$, with common bound $\leqq M$. Then for any complex number $\kappa$ with $|\kappa|<1 / M$, there exist $T(\kappa) \in \mathbb{S}_{+}(X)$ and $\hat{T}(\kappa) \in \mathbb{S}_{-}(X)$ uniquely determined by $(T, \hat{T}), A$ and $B$ with the following properties:

(3.1) $A$ is $(T(\kappa), \hat{T}(\kappa) ; p)$-smooth and $B^{\prime}$ is $\left(\hat{T}(\kappa)^{\prime}, T(\kappa)^{\prime} ; q\right)$-smooth with

$$
\|A\|_{(T(\kappa), \hat{T}(\kappa) ; p)} \leqq(1-|\kappa| M)^{-1}\|A\|_{(T, \hat{T} ; p)}
$$

and $\left\|B^{\prime}\right\|_{\left(\hat{T}\left(\kappa^{\prime}\right), T(\kappa)^{\prime} ; q\right)} \leqq(1-|\kappa| M)^{-1}\left\|B^{\prime}\right\|_{\left(\hat{T}^{\prime}, T^{\prime} ; q\right)}$.

(3.2) The pair $(T(\kappa), \hat{T}(\kappa)) \in \mathbb{B S}_{ \pm}(X)$.

(3.3) $T(\kappa) \supset T+\kappa B A, T \supset T(\kappa)-\kappa B A, \hat{T}(\kappa) \supset \hat{T}+\kappa B A$ and $\hat{T} \supset \hat{T}(\kappa)-\kappa B A$.

(3.4) $T(\kappa)$ is similar to $T$ and $\hat{T}(\kappa)$ is similar to $\hat{T}$.

More specifically, there exists a nonsingular operator $W(\kappa)$ in $\mathfrak{B}(X)$, given explicitly by

$$
\langle W(\kappa) u, v\rangle=\langle u, v\rangle-i \kappa \int_{0}^{\infty}\left\langle A e^{i s \hat{T}} u, B^{\prime} e^{-i s T(\kappa)^{\prime}} v\right\rangle d s, \quad u \in X, v \in X^{\prime}
$$

such that $T(\kappa)=W(\kappa) T W(\kappa)^{-1}$ and $\hat{T}(\kappa)=W(\kappa) \hat{T} W(\kappa)^{-1}$.

REMARK 3.2. If $T=\hat{T}$ and the operator $-i T$ generates a strongly continuous group $\left\{e^{-i t T}\right\}_{-\infty<t<\infty}$ of operators of $\mathfrak{B}(X)$ with type zero, then the perturbed 
operator $T(\kappa)=\hat{T}(\kappa)$ also generates a strongly continuous group of type zero. In this case two "wave operators" $W_{ \pm}(\kappa)$ exist and are given by

$$
\left\langle W_{ \pm}(\kappa) u, v\right\rangle=\left\langle u, v \pm i \kappa \int_{0}^{\infty}\left\langle A e^{\mp i t T} u, B^{\prime} e^{ \pm i t T(\kappa)^{\prime}} v ; d t\right.\right.
$$

(cf. Remark 4.13). These operators $W_{ \pm}(\kappa)$ coincide with the wave operators $\left({ }^{2}\right)$ $W^{ \pm}(\kappa)=\mathrm{s}-\lim _{t \rightarrow \pm \infty} e^{i t T(\kappa)} e^{-i t T}$ constructed according to the time-dependent scheme and furnish the intertwining relations

$$
T(\kappa)=W_{ \pm}(\kappa) T W_{ \pm}(\kappa)^{-1} .
$$

In particular, one can write down results for $X, Y$ Hilbert spaces and $p=q=2$. These agree with the work of Kato [6].

REMARK 3.3. We note that if an operator $A$ is $(T, \hat{T} ; p)$-smooth with respect to a pair $(T, \hat{T}) \in\left({ }^{\prime}{ }_{ \pm}(X)\right.$ and $T$ has a complete set of eigenvectors, then $A \equiv 0$. For if $A$ is $(T, \hat{T} ; p)$-smooth and $T u=\lambda u$, the integral (2.3) is unbounded unless $A u=0$. Thus our results are essentially concerned with the perturbation of continuous spectrum of $T$.

REMARK 3.4. An important and delicate part of this work involves the construction of the perturbed operator $T(\kappa)(\hat{T}(\kappa))$. We first construct the perturbed semigroup of operators $U(t, \kappa)(\hat{U}(t, \kappa))$ from the semigroups $e^{-i t T}, e^{i t \hat{T}}$ and the operators $A, B(\S 4.1)$. We next prove that the semigroups $U(t, \kappa)$ and $\hat{U}(t, \kappa)$ so obtained are strongly continuous of class $C_{0}$ with type zero. We denote by $-i T(\kappa)$ and $i \hat{T}(\kappa)$ their infinitesimal generators (they are closed and densely defined), respectively. We finally show that $T(\kappa) \supset T+\kappa B A$ and $\hat{T}(\kappa) \supset \hat{T}+\kappa B A(\S 4.2)$. This process is inevitable for general unbounded operators $A$ and $B$. In the case $B A \in \mathfrak{B}(X)$ we can set $T(\kappa)=T+\kappa B A$ and $\hat{T}(\kappa)=\hat{T}+\kappa B A$. Then most of the arguments in this paper can be greatly simplified.

REMARK 3.5. In the construction of $U(t, \kappa)(\hat{U}(t, \kappa))$, we use a formal perturbation series (cf. (4.5)). The smoothness assumptions on the operators $A$ and $B^{\prime}$ with respect to $(T, \hat{T})$ and $\left(\hat{T}^{\prime}, T^{\prime}\right)$, respectively, are needed to prove the absolute convergence of the perturbation series. It is worth while to point out that the successive approximations there appear to be generalizations of the interaction picture expansion

$$
U(t, \kappa)=U(t, 0)-i \kappa \int_{-\infty}^{t} e^{i(s-t) T} V e^{-i s T} d s+\cdots
$$

as used widely in Quantum Field Theory (cf. [21]).

REMARK 3.6. We also obtained some results by using the stationary method under a set of assumptions different from that of Theorem 3.1. This will be treated in a separate paper. We only mention here that our results by "time-dependent" and "stationary" methods are not related except for $p=q=2$; whereas in [6] they

${ }^{(2)}$ s-lim means strong limit. 
are nicely related by the Fourier-Plancherel theorem (cf. $[6, \S 3$, Lemma 3.6 and Theorem 3.9]).

\section{Proof of Theorem 3.1.}

1. Construction of the perturbed semigroups. Let $U_{0}(t)=e^{-i t T}$ be the unperturbed semigroup. For each fixed $u \in X$, set $S_{0}(t) u=A U_{0}(t) u$. Since $A$ is $(T, p,+)$-smooth, $S_{0}(t) u \in Y$ for almost all $t$ and, as a function of $t$, belongs to $\mathscr{Y}=L^{p}(0, \infty ; Y)$ with $\left\|S_{0}(\cdot) u\right\|_{\mathscr{g}} \leqq\|A\|_{(T, p,+)}\|u\|$.

Let us define

$$
S_{n}(\cdot) u=A e^{-i(\cdot) T} B \theta S_{n-1}(\cdot) u, \quad \text { for } n \geqq 1 .
$$

By our assumption, $A e^{-i(\cdot) T} B \theta \in \mathfrak{B}(\mathscr{Y})$. Thus it is clear that the $S_{n}(\cdot) u$ are well defined for each $u \in X$ and all $n \geqq 0$. Furthermore, $S_{n}(\cdot) u \in \mathscr{Y}$ with

$$
\left\|S_{n}(\cdot) u\right\|_{\mathscr{Y}} \leqq M^{n}\|A\|_{(T, p,+)}\|u\| .
$$

To construct our perturbed semigroup, we first define operators $\left\{U_{n}(t)\right\}$ as follows. For $0<t<\infty$ and $n \geqq 1$, define

$$
\left\langle U_{n}(t) u, v\right\rangle=\int_{0}^{t}\left\langle S_{n-1}(r) u, B^{\prime} e^{-i(t-r) T^{\prime}} v\right\rangle d r .
$$

To be more precise, for each $u \in X$ and $v \in X^{\prime}$, let us consider the following expression

$$
J_{n}(t, u, v)=\int_{0}^{t}\left\langle S_{n-1}(r) u, B^{\prime} e^{-i(t-r) T^{\prime}} v\right\rangle d r, \quad \text { for } n \geqq 1 .
$$

For each fixed $t$, the integral on the right exists and,

$$
\begin{aligned}
\left|J_{n}(t, u, v)\right| & \leqq\left(\int_{0}^{t}\left\|S_{n-1}(r) u\right\|^{p} d r\right)^{1 / p}\left(\int_{0}^{\infty}\left\|B^{\prime} e^{-i r T^{\prime}} v\right\|^{q} d r\right)^{1 / q} \\
& \leqq\left(\int_{0}^{t}\left\|S_{n-1}(r) u\right\|^{p} d r\right)^{1 / p}\left\|B^{\prime}\right\|_{\left(r^{\prime}, q_{,}+\right)}\|v\| .
\end{aligned}
$$

It follows that there exists an element $u_{t} \in X$ such that $\left\langle u_{t}, v\right\rangle=J_{n}(t, u, v)$. We define $U_{n}(t) u=u_{t}$. Clearly $U_{n}(t)$ belongs to $\mathfrak{B}(X)$ for all $t>0$ and

$$
\left\|U_{n}(t)\right\| \leqq\left\|S_{n-1}(\cdot) u\right\|_{\mathcal{B}}\left\|B^{\prime}\right\|_{\left(T^{\prime}, q,+\right)} \leqq M^{n-1}\|A\|_{(T, p,+)}\left\|B^{\prime}\right\|_{\left(T^{\prime}, q,+\right)}\|u\| .
$$

Moreover, $\lim _{t \backslash 0} U_{n}(t) u=0$. By setting $U_{n}(0)=0$, we thus obtained a family of bounded operators $\left\{U_{n}(t)\right\}_{0 \leqq t<\infty}, n \geqq 0$ of $\mathfrak{B}(X)$. Now we define

$$
U(t, \kappa) u=\sum_{n=0}^{\infty}(-i \kappa)^{n} U_{n}(t) u, \text { for } u \in X,|\kappa|<1 / M .
$$

Our goal is to show that $\{U(t, \kappa)\}_{0 \leqq t<\infty}$ is a strongly continuous semigroup of class $C_{0}$. To this end we shall first show that it is a semigroup. 
LEMMA 4.1. The family $\{U(t, \kappa)\}_{0 \leqq t<\infty}$ as defined in (4.5) is a semigroup of operators of $\mathfrak{B}(X)$, for every complex $\kappa$ with $|\kappa|<1 / M$.

Proof. Let us consider the series $\sum_{n=1}^{\infty}(-i \kappa)^{n} U_{n}(t)$. For each $u \in X$ we have, by (4.4),

$$
\begin{aligned}
\left\|\sum_{n=1}^{\infty}(-i \kappa)^{n} U_{n}(t) u\right\| & \leqq \sum_{n=1}^{\infty}|\kappa|^{n}\left\|U_{n}(t) u\right\| \\
& \leqq \sum_{n=1}^{\infty}|\kappa|^{n} M^{n-1}\|A\|_{(T, p,+)}\left\|B^{\prime}\right\|_{\left(T^{\prime}, q,+\right)}\|u\| \\
& \leqq|\kappa|\|A\|_{(T, p,+)}\left\|B^{\prime}\right\|_{\left(T^{\prime}, q,+\right)}\|u\|\left(\sum_{n=0}^{\infty}|\kappa|^{n} M^{n}\right) .
\end{aligned}
$$

Thus for any complex $\kappa$ with $|\kappa|<1 / M$, the series converges absolutely and uniformly with respect to $t$ for $0 \leqq t<\infty$. Since $U(t, \kappa)=U_{0}(t)+\sum_{n=1}^{\infty}(-i \kappa)^{n} U_{n}(t)$, it follows that $U(t, \kappa) \in \mathfrak{B}(X)$ for each $t, 0 \leqq t<\infty$. To see that $\{U(t, \kappa)\}_{0 \leqq t<\infty}$ does form a semigroup, first one observes that for each $u \in X, U_{n}(t) u \in D(A)$ for a.e. $t$., and that $A U_{n}(t) u=S_{n}(t) u$ as an element of $L^{p}(0, \infty ; Y)$. In fact, let $v \in D\left(A^{\prime}\right)$. By (4.1), (2.5) and noticing (4.3), we have, for a.e. $t$. and $n \geqq 1$,

$$
\begin{aligned}
\left\langle S_{n}(t) u, v\right\rangle & =\left\langle\left(A e^{-i(\cdot) T} B \theta S_{n-1}(\cdot) u\right)(t), v\right\rangle \\
& =\int_{0}^{t}\left\langle S_{n-1}(r) u, B^{\prime} e^{-i(t-r) T^{\prime}} A^{\prime} v\right\rangle d r \\
& =\left\langle U_{n}(t) u, A^{\prime} v\right\rangle .
\end{aligned}
$$

Since this is true for all $v \in D\left(A^{\prime}\right)$, it follows that $U_{n}(t) u$ belongs to $D\left(A^{\prime \prime}\right)=D(A)$ and $A U_{n}(t) u=S_{n}(t) u$ for a.e. $t$. Thus we can replace (4.3) by

$$
\left\langle U_{n}(t) u, v\right\rangle=\int_{0}^{t}\left\langle A U_{n-1}(r) u, B^{\prime} e^{-i(t-r) T^{\prime}} v\right\rangle d r,
$$

for $u \in X, v \in X^{\prime}, n \geqq 1$ and $0 \leqq t<\infty$.

Next we observe that

and

$$
U(t+s, \kappa)=\sum_{n=0}^{\infty}(-i \kappa)^{n} U_{n}(t+s)
$$

$$
\begin{aligned}
U(t, \kappa) U(s, \kappa) & =\left(\sum_{j=0}^{\infty}(-i \kappa)^{j} U_{j}(t)\right)\left(\sum_{k=0}^{\infty}(-i \kappa)^{k} U_{k}(s)\right) \\
& =\sum_{n=0}^{\infty}(-i \kappa)^{n}\left\{\sum_{j=0}^{n} U_{j}(t) U_{n-j}(s)\right\} .
\end{aligned}
$$

In order to show that $U(t+s, \kappa)=U(t, \kappa) U(s, \kappa)=U(s, \kappa) U(t, \kappa)$, by comparison of the coefficients of $(-i \kappa)^{n}$, it is enough to show that

$$
U_{n}(t+s)=\sum_{j=0}^{n} U_{j}(t) U_{n-j}(s)=\sum_{j=0}^{n} U_{j}(s) U_{n-j}(t) .
$$


These equalities can be shown easily by routine mathematical induction. The lemma follows.

It remains to show that the family $\{U(t, \kappa)\}_{0 \leqq t<\infty}$ is strongly continuous in $t$.

LEMMA 4.2. The semigroup $\{U(t, \kappa)\}_{0 \leqq t<\infty}$ is a strongly continuous semigroup of operators of $\mathfrak{B}(X)$ of class $C_{0}$ with type zero.

Proof. By definition, $U(t, \kappa)=\sum_{n=0}^{\infty}(-i \kappa)^{n} U_{n}(t)$; where $U_{0}(t)$ is the unperturbed semigroup which is strongly continuous in $t$. Let $t^{\prime}>t$. For $n \geqq 1, u \in X$ and $v \in X^{\prime}$ we have, by (4.3),

$$
\begin{aligned}
\left\langle U_{n}\left(t^{\prime}\right) u-U_{n}(t) u, v\right\rangle= & \int_{0}^{t^{\prime}}\left\langle S_{n-1}(r) u, B^{\prime} e^{-i\left(t^{\prime}-r\right) T^{\prime}} v\right\rangle d r \\
& -\int_{0}^{t}\left\langle S_{n-1}(r) u, B^{\prime} e^{-i(t-r) T^{\prime}} v\right\rangle d r \\
= & \int_{0}^{t}\left\langle S_{n-1}(r) u, B^{\prime}\left(e^{-i\left(t^{\prime}-r\right) T^{\prime}}-e^{-i(t-r) T^{\prime}}\right) v\right\rangle d r \\
& +\int_{t}^{t^{\prime}}\left\langle S_{n-1}(r) u, B^{\prime} e^{-i\left(t^{\prime}-r\right) T^{\prime}} v\right\rangle d r \\
= & J_{1}+J_{2} .
\end{aligned}
$$

Since $e^{-i\left(t^{\prime}-r\right) T^{\prime}}=e^{-i(t-r) T^{\prime}} e^{-i\left(t^{\prime}-t\right) T^{\prime}}$, we have

$$
\begin{aligned}
J_{1} & =\int_{0}^{t}\left\langle S_{n-1}(r) u, B^{\prime} e^{-i(t-r) T^{\prime}}\left(e^{-i\left(t^{\prime}-t\right) T^{\prime}}-I\right) v\right\rangle d r \\
& =\left\langle U_{n}(t) u,\left(e^{-i\left(t^{\prime}-t\right) T^{\prime}}-I\right) v\right\rangle \\
& =\left\langle\left(e^{-i\left(t^{\prime}-t\right) T}-I\right) U_{n}(t) u, v\right\rangle .
\end{aligned}
$$

Hence $\left|J_{1}\right| \leqq\left\|\left(e^{-i\left(t^{\prime}-t\right) T}-I\right) U_{n}(t) u\right\|\|v\|$.

$$
J_{2}=\int_{t}^{t^{\prime}}\left\langle S_{n-1}(r) u, B^{\prime} e^{-i\left(t^{\prime}-r\right) T^{\prime}} v\right\rangle d r
$$

We have

Consequently

$$
\left|J_{2}\right| \leqq\left(\int_{t}^{t^{\prime}}\left\|S_{n-1}(r) u\right\|^{p} d r\right)^{1 / p}\left\|B^{\prime}\right\|_{\left(T^{\prime}, q,+\right)}\|v\|
$$

$$
\begin{aligned}
\left\|U_{n}\left(t^{\prime}\right) u-U_{n}(t) u\right\| \leqq & \sup _{\|v\|=1}\left(\left|J_{1}\right|+\left|J_{2}\right|\right) \\
\leqq & \left\|\left(e^{-i\left(t^{\prime}-t\right) T}-I\right) U_{n}(t) u\right\| \\
& +\left\|B^{\prime}\right\|_{\left(T^{\prime}, q,+\right)}\left(\int_{t}^{t^{\prime}}\left\|S_{n-1}(r) u\right\|^{p} d r\right)^{1 / p} .
\end{aligned}
$$

Observe that in the right-hand side of the above inequality, the first term goes to zero as $t^{\prime} \rightarrow t$ due to the fact that $\left\{e^{-i t T}\right\}_{0 \leqq t<\infty}$ is of class $C_{0}$, while in the second term the function $g_{n-1}(r)=\left\|S_{n-1}(r) u\right\|^{p}$ is integrable over $(0, \infty)$ for each $n \geqq 1$, 
hence $\int g_{n-1}$ is absolutely continuous with respect to the Lebesgue measure on $(0, \infty)$, so $\int_{t}^{t^{\prime}} g_{n-1} \rightarrow 0$ as $t^{\prime} \rightarrow t$. Therefore we see that

$$
\left\|U_{n}\left(t^{\prime}\right) u-U_{n}(t) u\right\| \rightarrow 0 \text { as } t^{\prime} \rightarrow t, \text { for } t \geqq 0,
$$

i.e., $U_{n}(t)$ is strongly continuous in $t$ from the right for each $n \geqq 1$. Being the sum of $U_{0}(t)$ and $\sum_{n=1}^{\infty}(-i)^{n} U_{n}(t)$ (the series converges absolutely and uniformly with respect to $t$, cf. (4.6) and its proof), $U(t, \kappa)$ is therefore strongly continuous in $t$ from the right.

It is well known that one-sided strong continuity of a semigroup implies strong continuity. In fact, weak one-sided continuity of a semigroup is enough to insure its strong continuity (see Hille and Phillips [16, Theorem 10.2.3 and its corollary pp. 405-406]). Thus the family $\{U(t, \kappa)\}_{0 \leqq t<\infty}$ is a strongly continuous semigroup of operators of $\mathfrak{B}(X)$. It can be seen easily that $U(t, \kappa)$ is also of class $C_{0}$ with type zero. In fact, for each $u \in X$, let $f_{n}(t)=\sum_{m=1}^{n}(-i \kappa)^{m} U_{m}(t) u$ and $f(t)=\sum_{m=1}^{\infty}(-i \kappa)^{m}$ . $U_{m}(t) u$. Then, by (4.6) (Lemma 4.1), $f_{n} \rightarrow f$ uniformly in $(0, \infty)$. Since $f_{n}(t)$ are continuous functions of $t$ and $\lim _{t \rightarrow 0} f_{n}(t)=0$ for all $n \geqq 1$, thus

$$
\lim _{t \rightarrow 0} f(t)=\lim _{t \rightarrow 0} \lim _{n \rightarrow \infty} f_{n}(t)=\lim _{n \rightarrow \infty} \lim _{t \rightarrow 0} f_{n}(t)=0 .
$$

It follows that $\lim _{t \rightarrow 0} U(t, \kappa) u=\lim _{t \rightarrow 0} U_{0}(t) u+\lim _{t \rightarrow 0} f(t)=u$, because $U_{0}(t)$ is of class $C_{0}$. This implies that $U(t, \kappa)$ is also of class $C_{0}$. Moreover, for any $\varepsilon>0, t>0$,

$$
e^{-\varepsilon t}\|U(t, \kappa)\| \leqq e^{-\varepsilon t}\left\|U_{0}(t)\right\|+e^{-\varepsilon t}\left\|\sum_{n=1}^{\infty}(-i \kappa)^{n} U_{n}(t)\right\| .
$$

In this inequality, the first term on the right goes to zero as $t \rightarrow \infty$ due to the fact that $U_{0}(t)$ is of type zero. The same is true for the second term by (4.6). Hence $U(t, \kappa)$ is of type zero.

Having proved that $\{U(t, \kappa)\}_{0 \leqq t<\infty}$ is a strongly continuous semigroup of operators of $\mathfrak{B}(X)$ of class $C_{0}$ with type zero, let us denote by $-i T(\kappa)$ its infinitesimal generator. In what follows we shall write $e^{-i t T(\kappa)}$ for $U(t, \kappa)$.

REMARK 4.3. Throughout $\$ 4$.1, we may replace the operator $-i T$ by the operator $i \hat{T}$, and define $\hat{U}_{n}(t)$ by

$$
\begin{aligned}
\hat{U}_{0}(t) & =e^{i t \hat{T}} \\
\left\langle\hat{U}_{n}(t) u, v\right\rangle & =-\int_{0}^{t}\left\langle A \hat{U}_{n-1}(r) u, B^{\prime} e^{i(t-r) \hat{T}^{\prime}} v\right\rangle d r, \quad n \geqq 1, \\
\hat{U}(t, \kappa) & =\sum_{n=0}^{\infty}(-i \kappa)^{n} \hat{U}_{n}(t) .
\end{aligned}
$$

We can show similarly that $\{\hat{U}(t, \kappa)\}_{0 \leqq t<\infty}$ is a strongly continuous semigroup of operators of $\mathfrak{B}(X)$, for $|\kappa|<1 / M$. We shall denote by $i \hat{T}(\kappa)$ its infinitesimal generator and write $e^{i t \hat{T}(\kappa)}$ for $\hat{U}(t, \kappa)$.

2. Proof of (3.1) to (3.3). 
LEMMA 4.4. $A$ is $(T(\kappa), \hat{T}(\kappa) ; p)$-smooth and $B^{\prime}$ is $\left(\hat{T}(\kappa)^{\prime}, T(\kappa)^{\prime} ; q\right)$-smooth with

and

$$
\|A\|_{(T(\kappa), \hat{T}(\kappa) ; p)} \leqq(1-|\kappa| M)^{-1}\|A\|_{(T, \hat{T} ; p)}
$$

$$
\left\|B^{\prime}\right\|_{\left(\hat{T}(\kappa)^{\prime}, T(\kappa)^{\prime} ; q\right)} \leqq(1-|\kappa| M)^{-1}\left\|B^{\prime}\right\|_{\left(\hat{T}^{\prime}, T^{\prime} ; q\right)} .
$$

Proof. As was discussed in the proof of Lemma 4.1, for each $u \in X, U_{n}(t) u \in D(A)$ for almost all $t$ and we have $A U_{n}(t) u=S_{n}(t) u$ for all $n \geqq 0$. Let

$$
g_{n}(\cdot)=\sum_{m=0}^{n}(-i \kappa)^{m} A U_{m}(\cdot) u
$$

It follows, by (4.2), that

$$
\begin{aligned}
\sum_{n=0}^{\infty}\left\|(-i \kappa)^{n} A U_{n}(\cdot) u\right\|_{\mathscr{Y}} & =\sum_{n=0}^{\infty}\left\|(-i \kappa)^{n} S_{n}(\cdot) u\right\|_{\mathscr{Y}} \\
& \leqq \sum_{n=0}^{\infty}|\kappa|^{n} M^{n}\|A\|_{(T, p,+)}\|u\|=(1-|\kappa| M)^{-1}\|A\|_{(T, p,+)}\|u\|,
\end{aligned}
$$

for all $|\kappa|<1 / M$. Hence $g_{n}$ converges to an element $g \in \mathscr{Y}$. We can pick a subsequence $\left\{g_{n_{k}}\right\}$ of $\left\{g_{n}\right\}$ such that

$$
g_{n_{k}}(t) \rightarrow g(t), \text { for almost all } t .
$$

Since $\sum_{n=0}^{\infty}(-i \kappa)^{n} U_{n}(t) u$ converges uniformly in $t \in(0, \infty)$ (cf. Lemma 4.1, (4.6)) and $A$ is closed, it follows that, for almost all $t, \sum_{m=0}^{\infty}(-i \kappa)^{m} U_{m}(t) u \in D(A)$ and $A\left(\sum_{m=0}^{\infty}(-i \kappa)^{m} U_{m}(t) u\right)=\lim _{k \rightarrow \infty} g_{n_{k}}(t)=g(t)$. In other words, $e^{-i t T(x)} u \in D(A)$ for almost all $t$ and $A e^{-i t T(\kappa)} u=g(t)$. This implies that

$$
\left\|A e^{-i(\cdot) T(\kappa)} u\right\|_{\mathscr{Y}}=\|g\|_{\mathscr{Y}} \leqq(1-|\kappa| M)^{-1}\|A\|_{(T, p,+)}\|u\| .
$$

Hence $A$ is $(T(\kappa), p,+)$-smooth and

$$
\|A\|_{(T(\kappa), p,+)} \leqq(1-|\kappa| M)^{-1}\|A\|_{(T, p,+)} .
$$

The assertions that $A$ is $(\hat{T}(\kappa), p,-)$-smooth, $B^{\prime}$ is $\left(T(\kappa)^{\prime}, q,+\right)$-smooth and $B^{\prime}$ is $\left(\hat{T}(\kappa)^{\prime}, q,-\right)$-smooth can be proved similarly. This proves (3.1).

Before we go further, we need to investigate the adjoint of the convolution operator $C=A e^{-i(\cdot) T} B \theta \in \mathfrak{B}(\mathscr{Y})$. It is known (see Hille and Phillips [16, p. 89]) that the adjoint space of $\mathscr{Y}$ is $\mathscr{Y}^{\prime}=L^{q}\left(0, \infty ; Y^{\prime}\right)$. Hence we have $C^{\prime} \in \mathfrak{B}\left(\mathscr{Y}^{\prime}\right)$.

Let $[f, g]$ stand for the pairing between $f \in \mathscr{Y}$ and $g \in \mathscr{Y}^{\prime}$. Let $[a, b)$ be an arbitrary subinterval of $[0, \infty)$.

We define $\left({ }^{3}\right) f(s)=K_{[a, b)}(s) v, v \in D(B)$. Clearly $f \in \mathscr{Y}$. We have, by definition (see (2.5)), for any $w \in D\left(A^{\prime}\right)$,

$$
\begin{aligned}
\langle(C f)(\sigma), w\rangle & =\int_{0}^{\sigma}\left\langle f(\tau), B^{\prime} e^{-\imath(\sigma-\tau) T^{\prime}} A^{\prime} w\right\rangle d \tau \\
& =\int_{0}^{\sigma}\left\langle K_{[a, b)}(\tau) v, B^{\prime} e^{-i(\sigma-\tau) T^{\prime}} A^{\prime} w\right\rangle d \tau=J(\sigma) .
\end{aligned}
$$

$\left({ }^{3}\right) K_{[a, b)}$ stands for the characteristic function of $[a, b)$. 
If $\sigma<a$, we have $J(\sigma)=0$. For $\sigma \in[a, b)$

$$
J(\sigma)=\int_{a}^{\sigma}\left\langle v, B^{\prime} e^{-i(\sigma-\tau) T^{\prime}} A^{\prime} w\right\rangle d \tau=\int_{a}^{\sigma}\left\langle A e^{-i(\sigma-\tau) T} B v, w\right\rangle d \tau
$$

If $\sigma \geqq b$, then $J(\sigma)=\int_{a}^{b}\left\langle A e^{-i(\sigma-\tau) T} B v, w\right\rangle d \tau$. Since this is true for all $w \in D\left(A^{\prime}\right)$ which is dense in $Y^{\prime}$, thus we conclude that

$$
\begin{aligned}
(\boldsymbol{C f})(\sigma) & =0, & & \sigma<a \\
& =\int_{a}^{\sigma} A e^{-i(\sigma-\tau) T} B v d \tau, & & \sigma \in[a, b) \\
& =\int_{a}^{b} A e^{-i(\sigma-\tau) T} B v d \tau, & & \sigma \geqq b .
\end{aligned}
$$

The integrals are understood in the sense of Pettis. Thus for any $g \in \mathscr{Y}^{\prime}$, we have

$$
\begin{aligned}
{[C f, g]=} & \int_{0}^{\infty}\langle(C f)(\sigma), g(\sigma)\rangle d \sigma=\int_{0}^{a}+\int_{a}^{b}+\int_{b}^{\infty} \\
= & \int_{a}^{b} d \sigma \int_{a}^{\sigma}\left\langle A e^{-i(\sigma-\tau) T} B v, g(\sigma)\right\rangle d \tau \\
& +\int_{b}^{\infty} d \sigma \int_{a}^{b}\left\langle A e^{-i(\sigma-\tau) T} B v, g(\sigma)\right\rangle d \tau \\
= & \int_{a}^{b} d \tau\left\{\int_{\tau}^{\infty}\left\langle A e^{-i(\sigma-\tau) T} B v, g(\sigma)\right\rangle d \sigma\right\} .
\end{aligned}
$$

This change of the order of integration is justified. First we note that $\left\|A e^{-i t T} B v\right\|$ is measurable in $t$ implies that $\left\|A e^{-i(\sigma-\tau)} B v\right\|$ is measurable in $(\sigma, \tau)$. And

$$
\begin{aligned}
& \int_{a}^{b} \int_{\tau}^{\infty}\left|\left\langle A e^{-i(\sigma-\tau) T} B v, g(\sigma)\right\rangle\right| d \sigma d \tau \\
& \leqq\left(\int_{a}^{b} \int_{\tau}^{\infty}\left\|A e^{-i(\sigma-\tau) T} B v\right\|^{p} d \sigma d \tau\right)^{1 / p}\left(\int_{a}^{b} \int_{\tau}^{\infty}\|g(\sigma)\|^{q} d \sigma d \tau\right)^{1 / q} \\
& \leqq(b-a)^{1 / p}\|A\|_{(T, p,+)}\|B v\|(b-a)^{1 / q}\|g\|_{\mathscr{Y},} \\
& \leqq(b-a)\|A\|_{(T, p,+)}\|B v\|\|g\|_{\mathscr{Q},}<\infty
\end{aligned}
$$

Hence, Fubini's theorem can be applied.

On the other hand, $[C f, g]=\left[f, C^{\prime} g\right]=\int_{0}^{\infty}\left\langle f(\tau), C^{\prime} g(\tau)\right\rangle d \tau=\int_{a}^{b}\left\langle v, C^{\prime} g(\tau)\right\rangle d \tau$. Thus by combining the results above, we obtain

$$
\int_{a}^{b}\left\{\left\langle v, C^{\prime} g(\tau)\right\rangle-\int_{\tau}^{\infty}\left\langle A e^{-i(\sigma-\tau) T} B v, g(\sigma)\right\rangle d \sigma\right\} d \tau=0
$$


Since this holds for an arbitrary subset $[a, b)$ of $[0, \infty)$, it follows that the integrand vanishes almost everywhere in $[0, \infty)$, i.e. for a.e. $\tau$

$$
\left\langle v, C^{\prime} g(\tau)\right\rangle=\int_{\tau}^{\infty}\left\langle A e^{-i(\sigma-\tau) T} B v, g(\sigma)\right\rangle d \sigma .
$$

Similarly, for the operator $\hat{C}=A e^{i(\cdot) \hat{T}} B \theta$, we have

$$
\left\langle v, \hat{C}^{\prime} g(\tau)\right\rangle=\int_{\tau}^{\infty}\left\langle A e^{i(\sigma-\tau) \hat{T}} B v, g(\sigma)\right\rangle d \sigma,
$$

for all $v \in D(B)$ and $g \in \mathscr{Y}^{\prime}$.

For each fixed $t, 0<t<\infty$ and $v \in X^{\prime}$, let us define

and

$$
\begin{aligned}
g_{t}(r) & =B^{\prime} e^{-i(t-r) T^{\prime}} v, & & 0 \leqq r \leqq t \\
& =0, & & r>t ;
\end{aligned}
$$

$$
\begin{aligned}
U_{m, t}(r)^{\prime} v & =U_{m}(t-r)^{\prime} v, & & 0 \leqq r \leqq t \\
& =0, & & r>t,
\end{aligned}
$$

where $U_{m}(t), m \geqq 0$, are operators defined by (4.3). We note that $g_{t} \in \mathscr{Y}^{\prime}$.

LEMMA 4.5. $U_{m, t}(r)^{\prime} v \in D(B)$ for a.e. $r$ and

$$
B^{\prime} U_{m, t}(r)^{\prime} v=C^{\prime m} g_{t}(r), \text { for all } m \geqq 0 .
$$

Proof. Since $U_{0}(t)=e^{-i t T}$ and $B^{\prime}$ is $\left(T^{\prime}, q,+\right)$-smooth, we see that $B^{\prime} U_{0, t}(r)^{\prime} v$ $=B^{\prime} U_{0}(t-r)^{\prime} v=B^{\prime} e^{-i(t-r) T^{\prime}} v=g_{t}(r)$, thus (4.12) holds for $m=0$. Suppose that (4.12) holds for $m=n$. Let $w \in D(B)$, then

$\left\langle w, C^{\prime n+1} g_{t}(r)\right\rangle=\left\langle w, C^{\prime}\left(C^{\prime n} g_{t}\right)(r)\right\rangle=\int_{r}^{\infty}\left\langle A e^{-i(\sigma-\tau) r} B w, C^{\prime n} g_{t}(\sigma)\right\rangle d \sigma, \quad$ by (4.11).

But

$$
\int_{r}^{\infty}\left\langle A e^{-i(\sigma-r) T} B w, C^{\prime n} g_{t}(\sigma)\right\rangle d \sigma=\int_{0}^{\infty}\left\langle A e^{-i \sigma T} B w, C^{\prime n} g_{t}(\sigma+r)\right\rangle d \sigma,
$$

and, by inductive hypothesis, $C^{\prime n} g_{t}(\sigma+r)=B^{\prime} U_{n, t}(\sigma+r)^{\prime} v=B^{\prime} U_{n}(t-r-\sigma)^{\prime} v=$ $B^{\prime} U_{n, t-r}(\sigma)^{\prime} v=C^{\prime n} g_{t-r}(\sigma)$ (this makes sense for $r \leqq t$, and zero otherwise), and $A e^{-i \sigma T} B w=S_{0}(\sigma) B w$ (see the definition of $S_{0}(\cdot)$ ). It follows that the last integral becomes

$$
\begin{aligned}
\int_{0}^{\infty}\left\langle S_{0}(\sigma) B w, C^{\prime n} g_{t-r}(\sigma)\right\rangle d \sigma & =\left[S_{0}(\cdot) B w, C^{\prime n} g_{t-r}\right] \\
& =\left[C^{n} S_{0}(\cdot) B w, g_{t-r}\right]=\left[S_{n}(\cdot) B w, g_{t-r}\right] \\
& =\int_{0}^{t-r}\left\langle S_{n}(\sigma) B w, g_{t-r}(\sigma)\right\rangle d \sigma \\
& =\int_{0}^{t-r}\left\langle S_{n}(\sigma) B w, B^{\prime} e^{-i(t-r-\sigma) T^{\prime}} v\right\rangle d \sigma \\
& =\left\langle U_{n+1}(t-r) B w, v\right\rangle, \quad \text { by (4.3). }
\end{aligned}
$$


482

SUE-CHIN LIN

[May

Thus $\left\langle w, C^{\prime n+1} g_{t}(r)\right\rangle=\left\langle B w, U_{n+1}(t-r)^{\prime} v\right\rangle$, and hence

$$
C^{n+1} g_{t}(r)=B^{\prime} U_{n+1}(t-r)^{\prime} v=B^{\prime} U_{n+1, t}(r)^{\prime} v .
$$

Lemma 4.6. The semigroups $e^{-i t T}$ and $e^{-i t T(\kappa)}$ satisfy for $0 \leqq t<\infty$ the following relations: For $u \in X, v \in X^{\prime}$,

$$
\begin{aligned}
& \left\langle e^{-i t T(\kappa)} u-e^{-i t T} u, v\right\rangle=-i \kappa \int_{0}^{t}\left\langle A e^{-i r T(\kappa)} u, B^{\prime} e^{-i(t-r) T^{\prime}} v\right\rangle d r \\
& \left\langle e^{-i t T(\kappa)} u-e^{-i t T} u, v\right\rangle=-i \kappa \int_{0}^{t}\left\langle A e^{-i r T} u, B^{\prime} e^{-i(t-r) T(x)^{\prime}} v\right\rangle d r
\end{aligned}
$$

Proof. Formally we have

$$
\int_{0}^{t}\left\langle A e^{-r T(\kappa)} u, B^{\prime} e^{-i(t-r) T^{\prime}} v\right\rangle d r=\int_{0}^{t}\left\langle A\left(\sum_{n=0}^{\infty}(-i \kappa)^{n} U_{n}(r) u\right), B^{\prime} e^{-i(t-r) T^{\prime}} v\right\rangle d r .
$$

Recall that $A\left(\sum_{n=0}^{\infty}(-i \kappa)^{n} U_{n}(t) u\right)=\sum_{n=0}^{\infty}(-i \kappa)^{n} A U_{n}(t) u$ for almost all $t$ (cf. Lemma 4.4). Further, the sum

$$
\sum_{n=0}^{\infty}(-i \kappa)^{n} \int_{0}^{t}\left\langle A U_{n}(r) u, B^{\prime} e^{-i(t-r) T^{\prime}} v\right\rangle d r
$$

exists and equals to $\sum_{n=0}^{\infty}(-i \kappa)^{n}\left\langle U_{n+1}(t) u, v\right\rangle$. It follows that

$$
\int_{0}^{t}\left\langle A e^{-i r T(\kappa)} u, B^{\prime} e^{-i(t-r) T^{\prime}} v\right\rangle d r=(-i \kappa)^{-1}\left\langle\sum_{n=1}^{\infty}(-i \kappa)^{n} U_{n}(t) u, v\right\rangle .
$$

This implies that

$$
(-i \kappa) \int_{0}^{t}\left\langle A e^{-i r T(\kappa)} u, B^{\prime} e^{-i(t-r) T^{\prime}} v\right\rangle d r=\left\langle e^{-i t T(x)} u-e^{-i t T} u, v\right\rangle .
$$

(4.13) follows.

As for (4.14), first notice that

$$
\left\langle U_{n}(t) u, v\right\rangle=\int_{0}^{t}\left\langle S_{0}(r) u, B^{\prime} U_{n-1}(t-r)^{\prime} v\right\rangle d r, \quad n \geqq 1 .
$$

This can be seen easily by induction and Lemma 4.5 .

To prove (4.14), let us consider the expression

$$
\begin{aligned}
\int_{0}^{t}\left\langle A e^{-r T} u, B^{\prime} e^{-i(t-r) T(k)^{\prime}} v\right\rangle d r \\
=\int_{0}^{t}\left\langle A e^{-i r T} u, B^{\prime}\left(\sum_{n=0}^{\infty}(-i \kappa)^{n} U_{n}(t-r)^{\prime}\right) v\right\rangle d r \\
=\sum_{n=0}^{\infty}(-i \kappa)^{n} \int_{0}^{t}\left\langle A e^{-i r T} u, B^{\prime} U_{n}(t-r)^{\prime} v\right\rangle d r
\end{aligned}
$$


By (4.15), we see that the last quantity equals

$$
\begin{aligned}
\sum_{n=0}^{\infty}(-i \kappa)^{n}\left\langle U_{n+1}(t) u, v\right\rangle & =\left\langle\sum_{n=1}^{\infty}(-i \kappa)^{n} U_{n}(t) u, v\right\rangle \cdot(-i \kappa)^{-1} \\
& =\left\langle e^{-i t T(\kappa)} u-e^{-i t T} u, v\right\rangle \cdot(-i \kappa)^{-1}
\end{aligned}
$$

Thus (4.14) follows.

In the same fashion, we can prove

LEMMA 4.7. The semigroups $e^{i t \hat{T}}$ and $e^{i t \hat{T}(\kappa)}$ satisfy for $0 \leqq t<\infty$ the relations

$$
\begin{aligned}
& \left\langle e^{i t \hat{T}(\kappa)} u-e^{i t \hat{T}} u, v\right\rangle=i \kappa \int_{0}^{t}\left\langle A e^{i r \hat{f}(\kappa)} u, B^{\prime} e^{i(t-r) \hat{T}^{\prime}} v\right\rangle d r \\
& \left\langle e^{i t \hat{T}(\kappa)} u-e^{i t \hat{T}} u, v\right\rangle=i \kappa \int_{0}^{t}\left\langle A e^{i r \hat{T}} u, B^{\prime} e^{i(t-r) \hat{T}(\kappa)^{\prime}} v\right\rangle d r
\end{aligned}
$$

LEMMA 4.8. $T(\kappa) \supset T+\kappa B A, T \supset T(\kappa)-\kappa B A, \hat{T}(\kappa) \supset \hat{T}+\kappa B A$ and $\hat{T} \supset \hat{T}(\kappa)-\kappa B A$.

Proof. Let $R(z, \kappa)=(T(\kappa)-z)^{-1}$ and $R(z)=(T-z)^{-1}, z \in \Omega_{+}$. Now

$$
\begin{aligned}
i \int_{0}^{\infty} e^{i z t}\left[-i \kappa \int_{0}^{t}\left\langle A e^{-i r T(\kappa)} u,\right.\right. & \left.\left.B^{\prime} e^{-i(t-r) T^{\prime}} v\right\rangle d r\right] d t \\
& =\kappa \int_{0}^{\infty} d r\left[\int_{r}^{\infty} e^{i z t}\left\langle A e^{-i r T(\kappa)} u, B^{\prime} e^{-i(t-r) T^{\prime}} v\right\rangle d t\right] \\
& =\kappa \int_{0}^{\infty} d r\left[\int_{0}^{\infty} e^{i z(t+r)}\left\langle A e^{-i r T(\kappa)} u, B^{\prime} e^{-i t T^{\prime}} v\right\rangle d t\right] \\
& =\kappa \int_{0}^{\infty} d r e^{i z r}\left[\int_{0}^{\infty} e^{i z t}\left\langle A e^{-i r T(\kappa)} u, B^{\prime} e^{-i t T^{\prime}} v\right\rangle d t\right] \\
& =\kappa \int_{0}^{\infty} e^{i z r}\left\langle A e^{-i r T(\kappa)} u,-i B^{\prime} R(z)^{\prime} v\right\rangle d r \\
& =(-i)^{2} \kappa\left\langle A R(z, \kappa) u, B^{\prime} R(z)^{\prime} v\right\rangle \text { for } u \in X \text { and } v \in X^{\prime}
\end{aligned}
$$

(cf. Lemma 4.4 about the validity of the last steps). It follows that by taking the Laplace transform (except a constant factor) of both sides of (4.13), we obtain

$$
\begin{aligned}
\langle R(z, \kappa) u, v\rangle-\langle R(z) u, v\rangle & =-\kappa\left\langle A R(z, \kappa) u, B^{\prime} R(z)^{\prime} v\right\rangle \\
& =-\kappa\langle[R(z) B] A R(z, \kappa) u, v\rangle,
\end{aligned}
$$

where $[Q]$ denotes the closure of the operator $Q$. The fact that $[R(z) B]=B^{\prime} R(z)^{\prime}$ can be proved easily (cf. [19, Remark 1.8]). This implies that

(i) $R(z, \kappa)-R(z)=-\kappa[R(z) B] A R(z, \kappa)$.

Similarly, we have, by using (4.14), that

(ii) $R(z, \kappa)-R(z)=-\kappa[R(z, \kappa) B] A R(z)$.

To prove (3.3), let $u \in D(T) \cap D(B A)$ and $v=(T-z) u, z \in \Omega_{+}$. Then $R(z) v=u$, hence application of (ii) to $u$ gives $R(z, \kappa) v=u-\kappa[R(z, \kappa) B] A u=u-\kappa R(z, \kappa) B A u$. 
This implies that $u \in$ Range of $R(z, \kappa)=D(T(\kappa))$ and $(T(\kappa)-z) u=v+\kappa B A u=$ $(T-z) u+\kappa B A u=(T+\kappa B A) u-z u$. Hence $T(\kappa) \supset T+\kappa B A$. Similarly we can prove that $\hat{T}(\kappa) \supset \hat{T}+\kappa B A, \ldots$

It follows from Lemma 4.8 that $T(\kappa)$ is strictly equal to $T+\kappa B A$ and $\hat{T}(\kappa)$ is strictly equal to $\hat{T}+\kappa B A$, if $B A \in \mathfrak{B}(X)$.

Lemma 4.9. Let $T \in \mathscr{B S}_{+}(X)$ and $\hat{T} \in \mathscr{B S}_{-}(X)$. Then $T \subset \hat{T}$ if and only if $e^{i t \hat{T}} e^{-i t T}=I$ for all $0 \leqq t<\infty$.

Proof. Suppose $T \subset \hat{T}$. Let $Q(t)=e^{i t \hat{T}} e^{-i t T}$. Clearly $Q(t) \in \mathfrak{B}(X)$. Using thè facts that the function $Q(t) u$ is differentiable in $t$ for each $u \in D(T)$ and that $T \subset \hat{T}$, we get $Q(t) u=u$ for all $u \in D(T)$. Since $D(T)$ is dense in $X$ and $Q(t) \in \mathfrak{B}(X)$, we can therefore conclude that $Q(t)=I$ on $X$.

Conversely, suppose $Q(t)=I$ on $X$. Let $u \in D(T)$ and $v \in D\left(\hat{T}^{\prime}\right)$. We have $\langle u, v\rangle$ $=\langle Q(t) u, v\rangle=\left\langle e^{i t \hat{T}} e^{-i t T} u, v\right\rangle=\left\langle e^{-i t T} u, e^{i t \hat{T}^{\prime}} v\right\rangle$, by noting that $i \hat{T}^{\prime}$ also generates a strongly continuous semigroup. Now by differentiating both sides with respect to $t$ and evaluating at $t=0$, one gets $0=-i\langle T u, v\rangle+i\left\langle u, \hat{T}^{\prime} v\right\rangle$. This implies $\langle T u, v\rangle$ $=\left\langle u, \hat{T}^{\prime} v\right\rangle$ for every $v \in D\left(\hat{T}^{\prime}\right)$, thus $u \in D\left(\hat{T}^{\prime \prime}\right)=D(\hat{T})$ and $\hat{T} u=T u$. That is $T \subset \hat{T}$.

LEMMA 4.10. $e^{i t \hat{T}(\kappa)} e^{-i t T(\kappa)}=I$ for all $0 \leqq t<\infty$.

Proof. From our constructions, $e^{-i t T(\kappa)}=\sum_{n=0}^{\infty}(-i \kappa)^{n} U_{n}(t)$ and

$$
e^{i t \hat{T}(\kappa)}=\sum_{n=0}^{\infty}(-i \kappa)^{n} \hat{U}_{n}(t)
$$

Thus

$$
e^{i t \hat{T}(\kappa)} e^{-i t T(\kappa)}=\sum_{n=0}^{\infty}(-i \kappa)^{n} P_{n}(t),
$$

where $P_{n}(t)=\sum_{j=0}^{n} \hat{U}_{j}(t) U_{n-j}(t)$ for $n \geqq 0,0 \leqq t<\infty$. By using Lemma 4.9, equations (4.7), (4.8) and noting Remark 4.3 we can prove inductively that $P_{n}(t)=0$ for all $n \geqq 1$ and all $0 \leqq t<\infty$. (Although the proof is nontrivial and tricky, it is too long to be presented here; we omit it for aesthetic reasons, cf. [19, pp. 55-58].) We therefore conclude that

$$
e^{i t \hat{T}(\kappa)} e^{-i t T(\kappa)}=P_{0}(t)=I .
$$

Lemma 4.10 and Lemma 4.9 prove (3.2).

3. Defining formula for $W(\kappa)$ and proof of (3.4). For $u \in X$ and $v \in X^{\prime}$, the expression

$$
\langle W(\kappa) u, v\rangle=\langle u, v\rangle-i \kappa \int_{0}^{\infty}\left\langle A e^{i r} \hat{T} u, B^{\prime} e^{-i r T(\kappa)^{\prime}} v\right\rangle d r,
$$

defines a bounded operator $W(\kappa) \in \mathfrak{B}(X)$. For we have

$$
\begin{aligned}
\int_{0}^{\infty}\left\|A e^{i s \hat{T}} u\right\| \cdot\left\|B^{\prime} e^{-i s T(\kappa)^{\prime}} v\right\| d s & \leqq\left(\int\left\|A e^{i s \hat{T}} u\right\|^{p} d s\right)^{1 / p}\left(\int\left\|B^{\prime} e^{-i s T(\kappa)^{\prime}} v\right\|^{q} d s\right)^{1 / q} \\
& \leqq\|A\|_{(\hat{T}, p,-)}\|u\|\left\|B^{\prime}\right\|_{\left(T^{\prime}, q,+\right)}(1-|\kappa| M)^{-1}\|v\|
\end{aligned}
$$


(cf. Lemma 4.4). Let us write $W(\kappa)=I+\kappa P$, where

$$
\langle P u, v\rangle=-i \int_{0}^{\infty}\left\langle A e^{i s \hat{T}} u, B^{\prime} e^{-i s T(\kappa)^{\prime}} v\right\rangle d s .
$$

Similarly the expression

$$
\langle Z(\kappa) u, v\rangle=\langle u, v\rangle+i \kappa \int_{0}^{\infty}\left\langle A e^{i s \hat{T}(\kappa)} u, B^{\prime} e^{-i s T^{\prime}} v\right\rangle d s
$$

defines a $Z(\kappa) \in \mathfrak{B}(X)$. We write $Z(\kappa)=I+\kappa Q$, with

$$
\langle Q u, v\rangle=i \int_{0}^{\infty}\left\langle A e^{i s \hat{T}(\kappa)} u, B^{\prime} e^{-i s T^{\prime}} v\right\rangle d s .
$$

LEMMA 4.11. $W(\kappa) Z(\kappa)=Z(\kappa) W(\kappa)=I$.

Proof. Let $u \in X$ and $v \in X^{\prime}$ and we have

$$
\langle P Q u, v\rangle=\left\langle Q u, P^{\prime} v\right\rangle=i \int_{0}^{\infty}\left\langle A e^{i s \hat{T}(\kappa)} u, B^{\prime} e^{-i s T^{\prime}} P^{\prime} v\right\rangle d s
$$

Notice that for any $w \in D(B), v \in X^{\prime}$ one has

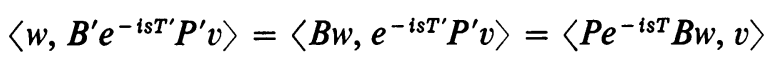

$$
\begin{aligned}
& =-i \int_{0}^{\infty}\left\langle A e^{i r \hat{T}} e^{-i s T} B w, B^{\prime} e^{-i r T(\kappa)^{\prime}} v\right\rangle d r \\
& =-i\left\{\int_{0}^{s}+\int_{s}^{\infty}\right\}
\end{aligned}
$$

The integrals inside \{\} can be evaluated separately as follows.

$$
\begin{aligned}
\int_{0}^{s} & =\int_{0}^{s}\left\langle A e^{-i(s-r) T} B w, B^{\prime} e^{-i r T(\kappa)^{\prime}} v\right\rangle d r \\
& =i / \kappa \cdot\left\langle e^{-i s T(\kappa)} B w-e^{-i s T} B w, v\right\rangle \\
& =i / \kappa \cdot\left\langle w, B^{\prime} e^{-i s T(\kappa)^{\prime}} v\right\rangle-i / \kappa\left\langle w, B^{\prime} e^{-i s T^{\prime}} v\right\rangle,
\end{aligned}
$$

for a.e. $s$, by using (4.13) and noting Lemma 4.9. And, by (4.11)^, we have

$$
\begin{aligned}
\int_{s}^{\infty} & =\int_{s}^{\infty}\left\langle A e^{i(r-s) \hat{T}} B w, B^{\prime} e^{-i r T(x)^{\prime}} v\right\rangle d r \\
& =\left\langle w, \hat{C}^{\prime} g(s)\right\rangle, \quad \text { where } g(r)=B^{\prime} e^{-i r T(x)^{\prime}} v
\end{aligned}
$$

The above results yield

$$
B^{\prime} e^{-i s T^{\prime}} P^{\prime} v=(1 / \kappa) \cdot B^{\prime} e^{-i s T(\kappa)^{\prime}} v-(1 / \kappa) \cdot B^{\prime} e^{-i s T^{\prime}} v-i \hat{C}^{\prime} g(s)
$$


for a.e. s. Hence

$$
\begin{aligned}
\langle P Q u, v\rangle= & i / \kappa \int_{0}^{\infty}\left\langle A e^{i s \hat{T}(\kappa)} u, B^{\prime} e^{-i s T(\kappa)^{\prime}} v\right\rangle d s \\
& -i / \kappa \cdot \int_{0}^{\infty}\left\langle A e^{i s \hat{T}(\kappa)} u, B^{\prime} e^{-i s T^{\prime}} v\right\rangle d s \\
& +\int_{0}^{\infty}\left\langle A e^{i s \hat{T}(\kappa)} u, \hat{C}^{\prime} g(s)\right\rangle d s \\
= & I_{1}+I_{2}+I_{3} .
\end{aligned}
$$

Let $f(s)=A e^{i s \hat{T}(x)} u(u$ fixed in $X)$, then

$$
\begin{aligned}
I_{3} & =\int_{0}^{\infty}\left\langle f(s), \hat{C}^{\prime} g(s)\right\rangle d s=\left[f, \hat{C}^{\prime} g\right]=[\hat{\boldsymbol{C}} f, g] \\
& =\int_{0}^{\infty}\langle\hat{\boldsymbol{C}} f(s), g(s)\rangle d s .
\end{aligned}
$$

Now for all $v \in D\left(A^{\prime}\right)$ and for a.e. $s$, we have, by $(2.5)^{\wedge}$

$$
\begin{aligned}
\langle\hat{C} f(s), v\rangle & =\int_{0}^{s}\left\langle f(r), B^{\prime} e^{i(s-r) \hat{T}^{\prime}} A^{\prime} v\right\rangle d r \\
& =\int_{0}^{s}\left\langle A e^{i r \hat{T}(x)} u, B^{\prime} e^{i(s-r) \hat{T}^{\prime}} A^{\prime} v\right\rangle d r .
\end{aligned}
$$

By $(4.13)^{\wedge}$, the last integral equals

$$
(1 / i \kappa)\left\langle e^{i s \hat{T}(\kappa)} u-e^{i s \hat{T}} u, A^{\prime} v\right\rangle=(1 / i \kappa)\left\langle A e^{i s \hat{T}(\kappa)} u, v\right\rangle-(1 / i \kappa)\left\langle A e^{i s \hat{T}} u, v\right\rangle
$$

for a.e. s. Hence

$$
\hat{C} f(s)=(1 / i \kappa) A e^{i s \hat{T}(\kappa)} u-(1 / i \kappa) A e^{i s \hat{T}} u, \text { for a.e. } s .
$$

It follows that

$$
\begin{aligned}
I_{3}= & \int_{0}^{\infty}(1 / i \kappa)\left\langle A e^{i s \hat{T}(\kappa)} u-A e^{i s \hat{T}} u, g(s)\right\rangle d s \\
= & (1 / i \kappa) \int_{0}^{\infty}\left\langle A e^{i s \hat{T}(\kappa)} u, B^{\prime} e^{-i s T(\kappa)^{\prime}} v\right\rangle d s \\
& -(1 / i \kappa) \int_{0}^{\infty}\left\langle A e^{i s \hat{T}} u, B^{\prime} e^{-i s T(\kappa)^{\prime}} v\right\rangle d s .
\end{aligned}
$$

The first term of $I_{3}$ cancels $I_{1}$, and the second term of $I_{3}$ is precisely equal to $(-1 / \kappa)\langle P u, v\rangle$. While $I_{2}=(-1 / \kappa)\langle Q u, v\rangle$. By combining $I_{1}, I_{2}$ and $I_{3}$, we obtain

$$
\langle P Q u, v\rangle=(-1 / \kappa)\langle Q u, v\rangle-(1 / \kappa)\langle P u, v\rangle .
$$

This implies $\kappa P Q+P+Q=0$, which is equivalent to $W(\kappa) Z(\kappa)=I$. The relation $Z(\kappa) W(\kappa)=I$ can be shown in the same manner. 
LEMMA 4.12. With the nonsingular operator $W(\kappa)$ constructed above, we have

(a) $W(\kappa) e^{-i r T}=e^{-i r T(\kappa)} W(\kappa)$ and

(b) $W(\kappa) e^{i r \hat{T}}=e^{i r \hat{T}(\kappa)} W(\kappa)$,

for all $r, 0 \leqq r<\infty$.

Proof. Let $u \in X$ and $v \in X^{\prime}$ and we have

$$
\left\langle W(\kappa) e^{-i r T} u, v\right\rangle=\left\langle e^{-i r T} u, v\right\rangle-i \kappa \int_{0}^{\infty}\left\langle A e^{i s \hat{T}} e^{-i r T} u, B^{\prime} e^{-i s T(\kappa)^{\prime}} v\right\rangle d s .
$$

The integral on the right-hand side can be split into two terms $\int_{0}^{r}$ and $\int_{r}^{s}$, where

$$
\begin{aligned}
-i \kappa \int_{0}^{r}\left\langle A e^{i s \hat{T}} e^{-i r T}\right. & \left.u, B^{\prime} e^{-i s T(\kappa)^{\prime}} v\right\rangle d s \\
& =-i \kappa \int_{0}^{r}\left\langle A e^{-i(r-s) T} u, B^{\prime} e^{-i s T(\kappa)^{\prime}} v\right\rangle d s \\
& =\left\langle e^{-i r T(\kappa)} u, v\right\rangle-\left\langle e^{-i r T} u, v\right\rangle,
\end{aligned}
$$

by noting Lemma 4.9 and using (4.14); and

$$
\begin{aligned}
-i \kappa \int_{r}^{\infty}\left\langle A e^{i s \hat{T}} e^{-i r T} u, B^{\prime} e^{-i s T(\kappa)^{\prime}} v\right\rangle d s \\
=-i \kappa \int_{r}^{\infty}\left\langle A e^{i(s-r) \hat{T}} u, B^{\prime} e^{-i s T(\kappa)^{\prime}} v\right\rangle d s \\
=-i \kappa \int_{0}^{\infty}\left\langle A e^{i s \hat{T}} u, B^{\prime} e^{-i s T(\kappa)^{\prime}} e^{-i r T(\kappa)^{\prime}} v\right\rangle d s
\end{aligned}
$$

by a change of variable $s-r \rightarrow r$. Consequently, by combining the above results, we get

$$
\begin{aligned}
\left\langle W(\kappa) e^{-i r T} u, v\right\rangle & =\left\langle u, e^{-i r T(\kappa)^{\prime}} v\right\rangle-i \kappa \int_{0}^{\infty}\left\langle A e^{i s \hat{T}} u, B^{\prime} e^{-i s T(\kappa)^{\prime}}\left(e^{-i r T(\kappa)^{\prime}} v\right)\right\rangle d s \\
& =\left\langle W(\kappa) u, e^{-i r T(\kappa)^{\prime}} v\right\rangle=\left\langle e^{-i r T(\kappa)} W(\kappa) u, v\right\rangle .
\end{aligned}
$$

Since this holds for all $u, v$, it follows that

$$
W(\kappa) e^{-i r T}=e^{-i r T(\kappa)} W(\kappa) .
$$

Similarly, one can prove (b). This gives the intertwining relations $W(\kappa) T$ $=T(\kappa) W(\kappa)$ and $W(\kappa) \hat{T}=\hat{T}(\kappa) W(\kappa)$.

The proof of the theorem is thus completed.

REMARK 4.13. In the case of the strongly continuous semigroups $\left\{e^{-i t T(x)}\right\}$ and $\left\{e^{i t \hat{T}(x)}\right\}_{0 \leqq t<\infty}$ constructed in $\S 4.1$, we are naturally interested in the investigation of the existence of the wave operators according to the time-dependent scheme. That is, we would like to know whether or not the strong limits of $W_{1}(t, \kappa)=e^{i t \hat{T}(\kappa)} e^{-i t T}$ and $W_{2}(t, \kappa)=e^{-i t T(\kappa)} e^{i t \hat{T}}$ exist as $t \rightarrow \infty$. It turns out that under the assumptions of our Theorem 3.1, the operator $W_{1}(\kappa)=s-\lim _{t \rightarrow \infty} W_{1}(t, \kappa)$ exists, but 
$\mathrm{s}-\lim _{t \rightarrow \infty} W_{2}(t, \kappa)$ does not seem to exist. Although $W_{1}(\kappa)$ exists, it does not give the intertwining relation between $T$ and $T(\kappa)$ or $\hat{T}$ and $\hat{T}(\kappa)$. However, in the special case $T=\hat{T}$, the roles of $T$ and $\hat{T}$ can be interchanged throughout the constructions of $T(\kappa)$ and $\hat{T}(\kappa)$; it follows that $T(\kappa)=\hat{T}(\kappa)$.

Thus $\left\{e^{-i t T(\kappa)}\right\}$ and $\left\{e^{-i t T}\right\}_{-\infty<t<\infty}$ are strongly continuous groups of operators of $\mathfrak{B}(X)$ with type zero. In this case, two "wave operators" $W_{ \pm}(\kappa)$ exist, given explicitly by

$$
\left\langle W_{ \pm}(\kappa) u, v\right\rangle=\langle u, v\rangle \pm i \kappa \int_{0}^{\infty}\left\langle A e^{\mp i t T} u, B^{\prime} e^{ \pm i t T(\kappa)^{\prime}} v\right\rangle d t,
$$

and we have

$$
T(\kappa)=W_{ \pm}(\kappa) T W_{ \pm}(\kappa)^{-1} .
$$

What is really interesting in this case is that the strong limits $W^{ \pm}(\kappa)$ of $W(t, \kappa)$ $=e^{i t T(\kappa)} e^{-i t T}$ as $t \rightarrow \pm \infty$ exist and coincide with $W_{ \pm}(\kappa)$. In fact, writing $e^{-i t T} u$ and $e^{i t T(x)^{\prime}} v$ for $u, v$ in (4.17), respectively, one gets

$$
\left\langle W_{+}(\kappa) e^{-i t T} u, e^{i t T(\kappa)^{\prime}} v\right\rangle=\langle W(t, \kappa) u, v\rangle+i \kappa \int_{0}^{\infty}\left\langle A e^{-i(t+r) T} u, B^{\prime} e^{i(t+r) T(\kappa)^{\prime}} v\right\rangle d r .
$$

Note that

$$
\begin{aligned}
\left\langle W_{+}(\kappa) e^{-i t T} u, e^{i t T(\kappa)^{\prime}} v\right\rangle & =\left\langle e^{i t T(\kappa)} W_{+}(\kappa) e^{-i t T} u, v\right\rangle \\
& =\left\langle W_{+}(\kappa) e^{i t T} e^{-i t T} u, v\right\rangle=\left\langle W_{+}(\kappa) u, v\right\rangle .
\end{aligned}
$$

Thus replacing $t+r$ by $r$ in the integral on the right, we get

$$
\left\langle W_{+}(\kappa) u, v\right\rangle=\langle W(t, \kappa) u, v\rangle+i \kappa \int_{t}^{\infty}\left\langle A e^{-i r T} u, B^{\prime} e^{i r T(\kappa)^{\prime}} v\right\rangle d r
$$

or

$$
\left\langle W_{+}(\kappa) u-W(t, \kappa) u, v\right\rangle=i \kappa \int_{t}^{\infty}\left\langle A e^{-i r T} u, B^{\prime} e^{i r T(\kappa)^{\prime}} v\right\rangle d r
$$

This implies

$$
\left|\left\langle W_{+}(\kappa) u-W(t, \kappa) u, v\right\rangle\right| \leqq|\kappa|\left(\int_{t}^{\infty}\left\|A e^{-i r T} u\right\|^{p} d r\right)^{1 / p}\left\|B^{\prime}\right\|_{\left(T(\kappa)^{\prime}, q,-\right)}\|v\| .
$$

It follows that

$$
\left\|W_{+}(\kappa) u-W(t, \kappa) u\right\| \leqq|\kappa|\left(\int_{t}^{\infty}\left\|A e^{-i r T} u\right\|^{p} d r\right)^{1 / p}\left\|B^{\prime}\right\|_{\left(T(\kappa)^{\prime}, q,-\right)} .
$$

By virtue of the assumption that

$$
\int_{0}^{\infty}\left\|A e^{-i r T} u\right\|^{p} d r<\infty
$$


the integral on the right of the above inequality (4.18) is finite and it therefore tends to zero as $t \rightarrow \infty$. This shows that $W(t, \kappa) \rightarrow W_{+}(\kappa)$ strongly as $t \rightarrow \infty$. The assertion about $W_{-}(\kappa)$ can be proved similarly.

5. Applications. In this section we shall apply our results to the perturbation problem involving the differential operator $-i d / d x$. We prove that Friedrichs' "gentle perturbation of second kind" can be handled by our method for general $p, 1<p<\infty$. Of course the results in this paper can also be applied to the examples studied by Kato [6, §6] about Schrödinger operator $L(\kappa)=-\Delta+\kappa f(\kappa)$ as well.

Let $X=L^{p}(0, \infty)$ and $Y=L^{p}((0, \infty) \times(0, \infty)), 1<p<\infty$. Let $D(\hat{T})=\{u \in X \mid u$ be absolutely continuous and its derivative $\left.u^{\prime} \in X\right\}$, and let $D(T)=\{u \in D(\hat{T}) \mid u(0)=0\}$. Define $\hat{T} u=-i u^{\prime}$ for $u \in D(\hat{T})$ and let $T$ be the restriction of $\hat{T}$ on $D(T)$. It is well known (cf. Dunford and Schwartz [20, pp. 629-630]) that $T \in\left(\mathfrak{S}_{+}(X)\right.$ and $\hat{T} \in \mathscr{S S}_{-}(X)$. Therefore the pair $(T, \hat{T}) \in \mathfrak{B S}_{ \pm}(X)$.

Let $k(x, y)$ be a complex valued measurable function on $(0, \infty) \times(0, \infty)$ such that

$$
\boldsymbol{\square} \rrbracket=\iint|k(x, y)| d x d y<\infty .
$$

Definition 5.1. Let $D(A)=\left\{u \in X: \iint|k(x, y)||u(y)|^{p} d x d y<\infty\right\}$ and let $D(B)$ $=\left\{v \in Y: \int\left[\int|k(x, y)|^{1 / q}|v(x, y)| d y\right]^{p} d x<\infty\right\}$, where $1 / p+1 / q=1$. Define $\left.{ }^{4}\right)$

$$
A u(x, y)=|k(x, y)|^{1 / p} u(y), \quad u \in D(A)
$$

and

$$
B v(x)=\int_{0}^{\infty} s(x, y)|k(x, y)|^{1 / q} v(x, y) d y, \quad v \in D(B),
$$

where $s(x, y)=\operatorname{sign} k(x, y)$.

We see that $A$ is an operator from $X$ to $Y$ and $B$ is an operator from $Y$ to $X$. If $u \in D(B A)$, then

$$
\begin{aligned}
B A u(x) & =\int s(x, y)|k(x, y)|^{1 / q} A u(x, y) d y \\
& =\int s(x, y)|k(x, y)|^{1 / q}|k(x, y)|^{1 / p} u(y) d y \\
& =\int k(x, y) u(y) d y .
\end{aligned}
$$

Thus the integral operator $V$ with kernel $k(x, y)$ is an extension of $B A$.

Lemma 5.2. $A \in \mathfrak{E}_{0}(X, Y)$ and $B \in \mathfrak{C}_{0}(Y, X)$.

Proof. $D(A)$ contains all bounded functions; in fact, if $|u(y)| \leqq M<\infty$, then $\iint|k(x, y)||u(y)|^{p} d x d y \leqq M^{p}|k|<\infty$. Thus $A$ is densely defined. To see that $A$ is

$\left(^{4}\right)$ We write $A u(x, y)$ for $(A u)(x, y)$ and $B v(x)$ for $(B v)(x)$ throughout this example. 
closed, let $u_{n} \in D(A)$ such that $u_{n} \rightarrow u$ in $X$ and $A u_{n} \rightarrow v$ in $Y\left({ }^{5}\right)$. Since $u_{n} \rightarrow u$ in $X$ $=L^{p}(0, \infty)$, there is a subsequence of $\left\{u_{n}\right\}$ which converges almost everywhere to $u$. For simplicity, we shall use the same index set for this subsequence; thus we have $u_{n}(y) \rightarrow u(y)$ for a.e. $y$. It follows that

$$
|k(x, y)|\left|u_{n}(y)\right|^{p} \rightarrow|k(x, y)||u(y)|^{p} \text { for a.e. } y .
$$

Since $\left\{|k(x, y)|\left|u_{n}(y)\right|^{p}\right\}$ is a sequence of nonnegative integrable functions, by Fatou's Lemma, we have

$$
\begin{aligned}
\iint|k(x, y)||u(y)|^{p} d x d y & \leqq \liminf _{n \rightarrow \infty} \iint|k(x, y)|\left|u_{n}(y)\right|^{p} d x d y \\
& =\liminf _{n \rightarrow \infty}\left\|A u_{n}\right\|^{p}=\|v\|^{p}<\infty .
\end{aligned}
$$

Thus $u \in D(A)$. Now that $A u_{n} \rightarrow v$ in $Y$, there is a subsequence $\left\{A u_{n_{k}}\right\}$ which converges to $v$ for a.e. $x, y$; but also we have $A u_{n_{k}}(x, y) \rightarrow A u(x, y)$ for a.e. $x, y$ (see (5.1)), consequently we must have $v(x, y)=A u(x, y)$ for a.e. $x, y$. This means that $A u=v$ in $Y$, hence $A$ is closed. Thus $A \in \mathfrak{\complement}_{0}(X, Y)$.

The adjoint operator $A^{\prime}$ of $A$ is given explicitly by

$$
A^{\prime} v(y)=\int_{0}^{\infty}|k(x, y)|^{1 / p} v(x, y) d x, \text { for } v \in D\left(A^{\prime}\right)
$$

where

$$
D\left(A^{\prime}\right)=\left\{v \in Y^{\prime}:\left.\left.\int_{0}^{\infty}\left|\int_{0}^{\infty}\right| k(x, y)\right|^{1 / p} v(x, y) d x\right|^{q} d y<\infty\right\} .
$$

This can be seen easily; in fact, if $v \in Y^{\prime}$ is such that

$$
\left.\left.\int_{0}^{\infty}\left|\int_{0}^{\infty}\right| k(x, y)\right|^{1 / p} v(x, y) d x\right|^{q} d y<\infty
$$

then for any $u \in D(A)$, we have

$$
\begin{aligned}
|\langle A u, v\rangle| & =\left.\left|\iint\right| k(x, y)\right|^{1 / p} u(y) v(x, y) d x d y \mid \\
& =\left|\int u(y)\left\{\int|k(x, y)|^{1 / p} v(x, y) d x\right\} d y\right| \\
& \leqq\|u\| \cdot\left\{\left.\left.\int\left|\int\right| k(x, y)\right|^{1 / p} v(x, y) d x\right|^{q} d y\right\}^{1 / q}<\infty .
\end{aligned}
$$

It follows that $v \in D\left(A^{\prime}\right)$. Conversely, if $v \in D\left(A^{\prime}\right)$, then $\left\langle u, A^{\prime} v\right\rangle=\langle A u, v\rangle$ for all $u \in D(A)$, i.e. $\left({ }^{6}\right)$.

$$
\int_{0}^{\infty} u(y)\left\{A^{\prime} v(y)-\int_{0}^{\infty}|k(x, y)|^{1 / p} v(x, y) d x\right\} d y=0 .
$$

(5) Convergence of vectors will always be strong convergence.

$\left.{ }^{(}\right)$Note that the change of the order of integration is justified. For

$$
\left.\iint|| k(x, y)\right|^{1 / p} v(x, y) \mid d x d y \leqq\|k\|^{1 / p}\|v\|<\infty .
$$


In particular, if $u(y)$ is the characteristic function of an arbitrary semiclosed interval $[a, b), 0 \leqq a<b<\infty$, then we have $\int_{a}^{b}\left\{A^{\prime} v(y)-\int_{0}^{\infty}|k(x, y)|^{1 / p} v(x, y) d x\right\} d y=0$. Since this is true for an arbitrary $[a, b)$, it follows that the integrand vanishes almost everywhere in $(0, \infty)$. That is,

$$
A^{\prime} v(y)=\int_{0}^{\infty}|k(x, y)|^{1 / p} v(x, y) d x, \text { for a.e. } y .
$$

Hence we have

$$
\left.\left.\int_{0}^{\infty}\left|\int_{0}^{\infty}\right| k(x, y)\right|^{1 / p} v(x, y) d x\right|^{q} d y<\infty
$$

As for the assertions about $B$, we can prove it indirectly as follows. Let

$$
D(F)=\left\{u \in X^{\prime}: \iint|k(x, y)||u(x)|^{q} d x d y<\infty\right\}
$$

We define

$$
F u(x, y)=|k(x, y)|^{1 / q} s(x, y) u(x), \quad u \in D(F) .
$$

Clearly $F$ is an operator from $X^{\prime}$ to $Y^{\prime}$. Just as we have proved that $A \in \mathfrak{C}_{0}(X, Y)$, we can show that $F \in \mathfrak{C}_{0}\left(X^{\prime}, Y^{\prime}\right)$. Hence $F^{\prime}$ exists and, furthermore, $D\left(F^{\prime}\right)$ is strongly dense in $Y$ (because $Y^{\prime}$ is reflexive, see Hille-Phillips [16, Theorem 2.11.9, p. 43]). Thus $F^{\prime} \in \mathfrak{C}_{0}(Y, X)$. By the same elementary computation as we did for $A^{\prime}$, we see that $F^{\prime}=B$. Hence $B \in \mathfrak{C}_{0}(Y, X)$.

TheOREM 5.3. Let $X=L^{p}(0, \infty)$ and $Y=L^{p}((0, \infty) \times(0, \infty)), 1<p<\infty$. Let $T, \hat{T}$ be as defined above. Let $k(x, y) \in L^{1}((0, \infty) \times(0, \infty))$ with $|k|=\int_{0}^{\infty} \int_{0}^{\infty}|k(x, y)| d x d y$, and let $A, B$ be operators defined in Definition 5.1 associated with the function $k(x, y)$. Then for all $|\kappa|<1 / \| k \rrbracket$, there exist $T(\kappa) \supset T+\kappa B A$ and $\hat{T}(\kappa) \supset \hat{T}+\kappa B A$ for which the results of Theorem 3.1 hold.

We shall build our proof on the following lemmas.

Lemma 5.4. $A$ is $(T, \hat{T} ; p)$-smooth and $B^{\prime}$ is $\left(\hat{T}, T^{\prime} ; q\right)$-smooth in the sense of Definitions 2.4 and 2.6, where $1<p<\infty$ and $1 / p+1 / q=1$.

Proof. The semigroup $U(t)=e^{-i t T}$ generated by $-i T$ and the semigroup $\hat{U}(t)$ $=e^{i t \hat{T}}$ generated by $i \hat{T}$ are, respectively, operators of right and left translation, i.e., for $u \in X$,

$$
\begin{aligned}
U(t) u(x) & =u(x-t), & & 0 \leqq t \leqq x \\
& =0, & & \text { otherwise; }
\end{aligned}
$$

and

$$
\hat{U}(t) u(x)=u(x+t), \quad 0 \leqq x
$$


Thus we have

$$
\begin{aligned}
\|A U(t) u\|^{p} & =\iint|A U(t) u(x, y)|^{p} d x d y \\
& =\int_{0}^{\infty} \int_{t}^{\infty}|k(x, y)||u(y-t)|^{p} d x d y
\end{aligned}
$$

hence

$$
\int_{0}^{\infty}\|A U(t) u\|^{p} d t=\int_{0}^{\infty} \int_{0}^{\infty}|k(x, y)| d x d y \int_{0}^{y}|u(y-t)|^{p} d t \leqq \llbracket k \rrbracket\|u\|^{p}
$$

and

$$
\|A \hat{U}(t) u\|^{p}=\iint|A \hat{U}(t) u(x, y)|^{p} d x d y=\iint|k(x, y)||u(y+t)|^{p} d x d y
$$

so that

$$
\int_{0}^{\infty}\|A \hat{U}(t) u\|^{p} d t=\iint|k(x, y)| d x d y \int|u(y+t)|^{p} d t \leqq\|k\|\|u\|^{p}
$$

It follows that $A$ is $(T, \hat{T} ; p)$-smooth with

$$
\|A\|_{(T, \hat{T} ; p)} \leqq \llbracket k \rrbracket
$$

The assertion that $B^{\prime}$ is $\left(\hat{T}^{\prime}, T^{\prime} ; q\right)$-smooth can be proved similarly, with the same estimate.

LEMma 5.5. $\left\|A e^{-i(\cdot) T} B \theta f\right\|_{\mathscr{S}} \leqq \llbracket k\|\| f \|_{\mathscr{Y}}$, and

$$
\left\|A e^{i(\cdot) \hat{T}} B \theta f\right\|_{\mathscr{Y}} \leqq \llbracket k\|\| f \|_{\mathscr{Y}}, \quad \text { for all } f \in \mathscr{Y}=L^{p}(0, \infty ; Y) .
$$

Proof. For any $f \in \mathscr{Y}, v \in X^{\prime}$ we have $F(t) \in X$ for every $t$ in $(0, \infty)$, where (cf. (2.5), Lemma 2.7)

$$
\langle F(t), v\rangle=\int_{0}^{t}\left\langle f(s), B^{\prime} e^{-i(t-s) T^{\prime}} v\right\rangle d s
$$

We must show that $F(t) \in D(A)$ for a.e. $t$ and, as a function of $t, A F(t) \in \mathscr{Y}$, that is, $\int_{0}^{\infty}\|A F(t)\|^{p} d t<\infty\left({ }^{7}\right)$.

Let $v \in D\left(A^{\prime}\right)$. By (2.5), we have

$$
\langle A F(t), v\rangle=\left\langle F(t), A^{\prime} v\right\rangle=\int_{0}^{t}\left\langle f(s), B^{\prime} e^{-i(t-s) T^{\prime}} A^{\prime} v\right\rangle d s .
$$

( $\left.{ }^{7}\right)$ Again the extended norm is used (cf. Definition 2.2). 
Hence

$$
\begin{aligned}
|\langle A F(t), v\rangle| & \leqq \int_{0}^{t}\left|\left\langle f(s), B^{\prime} e^{-i(t-s) T^{\prime}} A^{\prime} v\right\rangle\right| d s \\
& \leqq \int_{0}^{t} \int_{0}^{\infty} \int_{0}^{\infty}|f(s, x, y)|\left|B^{\prime} e^{-i(t-s) T^{\prime}} A^{\prime} v(x, y)\right| d x d y d s \\
& =\iiint|f(s, x, y)||k(x, y)|^{1 / a} A^{\prime} v(x-t+s) d x d y d s
\end{aligned}
$$

by noting that $\left|B^{\prime} g(x, y)\right|=|k(x, y)|^{1 / q}|g(x)|$ (cf. (5.3)).

However, since $A^{\prime} v(x-t+s)=\int_{0}^{\infty}|k(x-t+s, \sigma)|^{1 / p} v(x-t+s, \sigma) d \sigma$ by (5.2), we thus have

$$
\begin{aligned}
|\langle A F(t), v\rangle| & \\
\leqq & \iiint \int\left(|f(s, x, y)||k(x-t+s, \sigma)|^{1 / p}\right)\left(|k(x, y)|^{1 / q}|v(x-t+s, \sigma)|\right) d x d y d s d \sigma \\
\leqq & \left\{\iiint \int|f(s, x, y)|^{p}|k(x-t+s, \sigma)| d x d y d s d \sigma\right\}^{1 / p} \\
& \cdot\left\{\iiint \int|k(x, y)||v(x-t+s, \sigma)|^{q} d x d y d s d \sigma\right\}^{1 / q} \leqq \alpha(t) \mid k \mathbf{|}^{1 / q}\|v\|,
\end{aligned}
$$

where

$$
\alpha(t)=\left\{\iiint \int|f(s, x, y)|^{p}|k(x-t+s, \sigma)| d x d y d s d \sigma\right\}^{1 / p} .
$$

Consequently

$$
\|A F(t)\| \leqq \alpha(t) \boldsymbol{k}^{\mathbf{1 / q}}
$$

Since

$$
\begin{aligned}
\int_{0}^{\infty} \alpha(t)^{p} d t & =\iiint \iint|f(s, x, y)|^{p}|k(x-t+s, \sigma)| d x d y d s d \sigma d t \\
& \leqq \llbracket k\|f\|_{\mathscr{W}}^{p},
\end{aligned}
$$

it follows that

$$
\int_{0}^{\infty}\|A F(t)\|^{p} d t \leqq \llbracket k \rrbracket^{p / q} \int_{0}^{\infty} \alpha(t)^{p} d t \leqq \llbracket k \mathbf{\square}^{1+p / q}\|f\|_{\mathscr{Q}}^{p}
$$

This proves that $f \in D\left(A e^{-i(\cdot) T} B \theta\right)$ and $\left\|A e^{-i(\cdot) T} B \theta f\right\|_{\mathscr{Y}}=\|A F(\cdot)\|_{\mathscr{Y}} \leqq \llbracket k \rrbracket\|f\|_{\mathscr{Y}}$. The second assertion can be proved in the same way with the same bound.

Theorem 5.3 follows from Lemmas 5.2, 5.4 and 5.5.

REMARK 5.6. We may consider the differential operator $T=-i d / d x$ acting in $L^{p}(-\infty, \infty)$ instead of $L^{p}(0, \infty)$ throughout Theorem 5.3, and the kernel function $k(x, y)$ of the disturbances defined on $-\infty<x, y<\infty$ instead of $0 \leqq x, y<\infty$. It is easy to see that our whole process can be applied to this case without any significant 
change (in fact it is even simpler, for we can, in this case, simply take $T=\hat{T}$ ). Consequently Friedrichs' "gentle perturbation of second kind" can be handled by our method completely.

\section{BIBLIOGRAPHY}

1. K. O. Friedrichs, Über die Spektralzerlegung eines Integral-operators, Math. Ann. 115 (1938), 259-272.

2. - On the perturbation of continuous spectra, Comm. Pure Appl. Math. 1 (1948), 361-406.

3. M. Rosenblum, Perturbation of the continuous spectrum and unitary equivalence, Pacific $\mathbf{J}$. Math. 7 (1957), 997-1010.

4. T. Kato, On finite-dimensional perturbations of self-adjoint operators, J. Math. Soc. Japan 9 (1957), 239-249.

5. - Perturbation of continuous spectra by trace class operators, Proc. Japan Acad. 33 (1957), 260-264.

6. - Wave operators and similarity for some non-selfadjoint operators, Math. Ann. 162 (1966), 258-279.

7. O. A. Ladyženskaja and L. D. Fadeev, On the perturbation of continuous spectra, Dokl. Akad. Nauk SSSR 120 (1958), 1187-1190.

8. J. Schwartz, Some non-self-adjoint operators, Comm. Pure Appl. Math. 13 (1960), 609-639.

9. S. T. Kuroda, Perturbation of continuous spectra by unbounded operators. I, II, J. Math. Soc. Japan 11 (1959), 247-262; 12 (1960), 243-257.

10. - An abstract stationary approach to perturbation of continuous spectra and scattering theory, J. Analyse Math. 20 (1967), 57-117.

11. L. de Branges, Perturbations of self-adjoint transformations, Amer. J. Math. 84 (1962), 543-560.

12. P. A. Rejto, On gentle perturbations. I, II, Comm. Pure Appl. Math. 16 (1963), 279-303; 17 (1964), 257-292.

13. M. S. Birman and M. G. Kreĭn, On the theory of wave operators and scattering operators, Dokl. Akad. Nauk SSSR 144 (1962), 475-478=Soviet Math. Dokl. 3 (1962), 740-744.

14. M. S. Birman and S. B. Èntina, A stationary approach in the abstract theory of scattering, Dokl. Akad. Nauk SSSR 155 (1964), 506-508= Soviet Math. Dokl. 5 (1964), 432-434.

15. K. O. Friedrichs, Perturbation of spectra in Hilbert space, Lectures in Appl. Math., Vol. 3, Amer. Math. Soc. Providence, R. I., 1965.

16. E. Hille and R. S. Phillips, Functional analysis and semi-groups, Amer. Math. Soc. Colloq. Publ., Vol. 31, Amer. Math. Soc., Providence, R. I., 1957.

17. T. Kato, Perturbation theory for linear operators, Springer-Verlag, New York, 1966.

18. P. R. Halmos, Measure theory, Van Nostrand, Princeton, N. J., 1962.

19. S. C. Lin, Wave operators and similarity for some operators in Banach spaces, Ph.D. thesis, Univ. of Calif., Berkeley, 1967.

20. N. Dunford and J. T. Schwartz, Linear operators, Part I, Interscience, New York, 1958.

21. R. T. Prosser, Convergent perturbation expansions for certain wave operators, J. Math. Phys. 5 (1964), 708-713.

\footnotetext{
UNIVERSITY OF CALIFORNIA, BERKELEY, CALIFORNIA

UNIVERSITY OF MIAMI,

Coral Gables, Florida
} 